\title{
PRYMS OF NON-CYCLIC TRIPLE COVERINGS AND LOG CANONICAL MODELS OF THE SPIN MODULI SPACE OF GENUS 2
}

\author{
HERBERT LANGE AND ANGELA ORTEGA
}

\begin{abstract}
We show that the moduli spaces of non-cyclic triple covers of genus 2 curves and that of even spin curves of genus 2 are birationally isomorphic via the Prym map. We describe the $\log$ canonical models of the moduli space of $S_{2}^{+}$and use this to extend the above birational map.
\end{abstract}

\section{INTRODUCTION}

In recent years, the log minimal model program has been carried out completely for the moduli space $\bar{M}_{g}$ in low genera and in many cases these models have also a modular meaning. For instance, in [16] the Deligne-Mumford compactification and the GIT compactification of $M_{2}$ are realized as log minimal models of $\bar{M}_{2}$. In this paper we study the $\log$ canonical models of the moduli space $\bar{S}_{2}^{+}$of even spin curves of genus 2. Our initial motivation was to find out if the birational morphism, induced by the Prym map, between the moduli space $R_{2,3}^{n c}$ of non-cyclic étale triple coverings of genus 2 curves and $S_{2}^{+}$([22], [23]) could be related to a new modular compactification of the spin moduli space. In order to state our theorems let us recall some previous results.

Let $f: Y \rightarrow X$ be a non-cyclic étale 3-fold covering of a smooth projective curve $X$ of genus 2. Its Prym variety $P(f):=(\operatorname{Ker} N m f)^{0}$ is a Jacobian surface with principal polarization $\Xi$, giving rise to a map $\operatorname{Pr}: R_{2,3}^{n c} \rightarrow \mathcal{J}_{2}$ into the moduli space of canonically polarized Jacobian surfaces. In [22] we showed that this map is finite of degree 10 onto its image which is an open set in $\mathcal{J}_{2}$. We also proved that the Prym map is not surjective and determined its image explicitly.

In [23] we extended this map to a proper map which is surjective onto the moduli space $\mathcal{A}_{2}$ of principally polarized abelian surfaces. To be more precise, we denote by $\mathfrak{S}_{3}:=\left\langle\sigma, \tau \mid \sigma^{3}=\tau^{2}=(\sigma \tau)^{2}=1\right\rangle$ the symmetric group of order 6 and let $\mathfrak{S}_{3} \bar{M}_{2}$ denote the moduli space of admissible $\mathcal{S}_{3}$-covers of stable curves of genus 2 , as defined in [1]. We consider the following open subspace

$$
\mathfrak{S}_{3} \widetilde{M}_{2}:=\left\{\begin{array}{l|l}
{[h: Z \rightarrow X] \in \mathfrak{S}_{3} \bar{M}_{2}} & \begin{array}{l}
p_{a}(Z)=7 \text { and for any node } z \in Z \\
\text { the stabilizer } \operatorname{Stab}(z) \text { is of order 3 }
\end{array}
\end{array}\right\} .
$$

For all $[h: Z \rightarrow X] \in \mathfrak{S}_{3} \widetilde{M}_{2}$ the Prym variety of $f: Y:=Z /\langle\tau\rangle \rightarrow X$ is a principally polarized abelian surface, independent of the involution $\tau \in \mathfrak{S}_{3}$. This defines a Prym map $\operatorname{Pr}: \mathfrak{S}_{3} \widetilde{M}_{2} \rightarrow \mathcal{A}_{2}$ which is proper, surjective and finite of degree 10 .

1991 Mathematics Subject Classification. 14H10, 14H30, 14H40.

Key words and phrases. Prym variety, Prym map, spin curve.

The second author was supported by Deutsche Forschungsgemeinschaft, SFB 647 . 
The description of the fibres of the Prym map suggests that there is a close relation between the space $\mathfrak{S}_{3} \widetilde{M}_{2}$ and the moduli space $S_{2}^{+}$of even spin curves of genus 2 . We define the following open subspace

$$
\mathfrak{S}_{3} \widehat{M}_{2}:=\left\{[h: Z \rightarrow X] \in \mathfrak{S}_{3} \widetilde{M}_{2} \mid \mathrm{X} \text { irreducible }\right\} .
$$

Then our first result is

Theorem 1. There is a canonical isomorphism $\alpha: \mathfrak{S}_{3} \widehat{M}_{2} \rightarrow S_{2}^{+}$.

This is true even on the level of moduli stacks (see Theorem 3.1). We then consider the question whether one can extend this isomorphism to a regular map defined on the whole of $\mathfrak{S}_{3} \widetilde{M}_{2}$ with values in some compactification of $S_{2}^{+}$. For this we work out the log canonical models of the compactification $\bar{S}_{2}^{+}$of $S_{2}^{+}$, as defined by Cornalba in [8]. We denote by $\overline{\mathcal{S}}_{2}^{+}$(respectively $\mathcal{S}_{2}^{+}$) the moduli stack corresponding to $\bar{S}_{2}^{+}$(respectively $S_{2}^{+}$) and by $\delta:=\overline{\mathcal{S}}_{2}^{+} \backslash \mathcal{S}_{2}^{+}$the boundary divisor. The log canonical models of $\overline{\mathcal{S}}_{2}^{+}$with respect to $K_{\overline{\mathcal{S}}_{2}^{+}}+\epsilon \delta$ are defined by

$$
\overline{\mathcal{S}}_{2}^{+}(\epsilon):=\operatorname{Proj}\left(\bigoplus_{n \geq 0} \Gamma\left(\overline{\mathcal{S}}_{2}^{+}, n\left(K_{\overline{\mathcal{S}}_{2}^{+}}+\epsilon \delta\right)\right)\right),
$$

for $\epsilon \in \mathbb{Q} \cap[0,1]$. The fact that sections of invertible sheaves on $\overline{\mathcal{S}}_{2}^{+}$are pullbacks of sections of the corresponding sheaves on $\bar{S}_{2}^{+}$implies that the log canonical model of $\overline{\mathcal{S}}_{2}^{+}$ with respect to $K_{\overline{\mathcal{S}}_{2}^{+}}+\epsilon \delta$ can be identified with the log canonical model $\bar{S}_{2}^{+}(\epsilon)$ of $\bar{S}_{2}^{+}$with respect to the corresponding divisor in $\bar{S}_{2}^{+}$(see Corollary 6.4).

The following theorem (see the end of Sections 6 and 9) is analogous to the corresponding result of Hassett's for $\bar{M}_{2}$ (see [16, Theorem 4.10]).

Theorem 2. Consider the log canonical model of $\overline{\mathcal{S}}_{2}^{+}$with respect to $K_{\overline{\mathcal{S}}_{2}^{+}}+\epsilon \delta$, that is, the log canonical model of $\bar{S}_{2}^{+}$with respect to the corresponding divisor in $\bar{S}_{2}^{+}$.

(1) For $\epsilon>\frac{57}{25}$ we have $\bar{S}_{2}^{+}(\epsilon) \simeq \bar{S}_{2}^{+}$.

(2) For $\frac{49}{25}<\epsilon \leq \frac{57}{25}$ we have $\bar{S}_{2}^{+}(\epsilon) \simeq{\overline{S_{2}^{+}}}^{i n v}$, where ${\overline{S_{2}^{+}}}^{i n v}$ denotes the invariant theoretical compactification (for the definition see Section 7).

(3) For $\epsilon=\frac{49}{25}$ we get a point; the log canonical divisor fails to be effective for $\epsilon<\frac{49}{25}$.

The invariant theoretical compactification of $S_{2}^{+}$is the Proj of certain ring of invariants arising from the parametrization of binary sextic forms together with a partition of the roots into two sets of three elements. Concerning the extension of the map $\alpha$ to the whole of $\mathfrak{S}_{3} \widetilde{M}_{2}$ we finally obtain (see Propositions 4.2 and 10.1):

\section{Theorem 3.}

(1) The construction defining the isomorphism $\alpha: \mathfrak{S}_{3} \widehat{M}_{2} \rightarrow S_{2}^{+}$does not extend to $\mathfrak{S}_{3} \widetilde{M}_{2}$. 


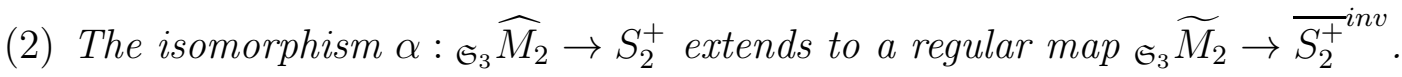

The paper is organized as follows. In Section 2 we recall the definitions of the main moduli stacks and spaces which are used in the sequel. In Section 2 the proof of Theorem 1 is given. In Section 4 we show Theorem 3 (1). In Section 5 we compute the nef cone of $\bar{S}_{2}^{+}$and apply this in Section 6 to prove Theorem 2 (1). In Section 7 and 8 we study the invariant-theoretical compactification ${\overline{S_{2}^{+}}}^{i n v}$ and the canonical map $\bar{S}_{2}^{+} \rightarrow{\overline{S_{2}^{+}}}^{i n v}$ and use this in Section 9 to give a proof of Theorem 2 (2) and 2 (3). Finally, in Section 10 we prove Theorem 3 (2).

We work over an algebrically closed field $k$ of characteristic zero. Moduli spaces are denoted by capital bold letters, the corresponding moduli stacks by the corresponding cursive letters. Divisors on a coarse moduli space are denoted by capital Latin letters, divisors on a moduli stack by small Greek letters. We denote a divisor and its class in the rational Picard group by the same letter.

We thank I. Dolgachev for the hint leading to Remark 7.2, The second author is thankful to G. Farkas for helpful and stimulating discussions.

\section{THE MODULI STACKS}

2.1. The stacks of $\mathfrak{S}_{3}$-coverings of genus-2 curves. Let $\mathfrak{S}_{3} \mathcal{M}_{2}$ denote the moduli stack of étale Galois covers of smooth curves of genus 2 with Galois group the symmetric group $\mathfrak{S}_{3}$ of order 6 ([2, Theorem 17.2.11]) and its compactification $\mathfrak{S}_{3} \overline{\mathcal{M}}_{2}$ of ${ }_{\mathfrak{S}_{3}} \mathcal{M}_{2}$ by admissible $\mathfrak{S}_{3}$-covers as constructed in [1] (see also [2, Chapter 17]).

We consider the following open substack ${ }_{\mathfrak{S}_{3}} \widehat{\mathcal{M}}_{2}$ of $\mathfrak{S}_{3} \overline{\mathcal{M}}_{2}$ associated to the functor $F: S c h / \mathbb{C} \rightarrow$ Ens defined by

$$
S \mapsto\left\{\begin{array}{l|l}
{[h: \mathcal{Z} \rightarrow \mathcal{X} \text { over } S] \in \mathfrak{S}_{3} \overline{\mathcal{M}}_{2}} & \begin{array}{l}
\text { for all } s \in S, p_{a}\left(\mathcal{Z}_{s}\right)=7, \mathcal{X}_{s} \text { is irreducible, } \\
\text { for any node } z \in \mathcal{Z}_{s}, \text { Stab }(z) \text { is of order } 3
\end{array}
\end{array}\right\} .
$$

Note that $\mathfrak{S}_{3} \widehat{\mathcal{M}}_{2}$ is a smooth Deligne-Mumford stack of dimension 3. The functor $F$ admits a coarse moduli space denoted by $\mathfrak{S}_{3} \widehat{M}_{2}$.

According to 23 the stack admits a stratification

$$
\mathfrak{S}_{3} \widehat{\mathcal{M}}_{2}=\mathfrak{S}_{3} \mathcal{M}_{2} \sqcup \mathcal{R}_{2} \sqcup \mathcal{R}_{1}
$$

Here $\mathcal{R}_{2}$ (respectively $\mathcal{R}_{1}$ ) denotes the locally closed substack of $\mathfrak{S}_{3} \widehat{\mathcal{M}}_{2}$ where $\mathcal{X}_{s}$ admits exactly one node (respectively two nodes) for all $s$. The index refers to the dimension of the substack. Similarly, there is a stratification $\mathfrak{S}_{3} \widehat{M}_{2}=\mathfrak{S}_{3} M_{2} \sqcup R_{2} \sqcup R_{1}$ for the corresponding moduli spaces.

2.2. The stacks of genus-2 spin curves. Recall that a smooth spin curve of genus 2 is a pair $(C, \kappa)$ with $C$ a smooth curve of genus 2 and $\kappa$ a theta characteristic on $C$, i.e. a line bundle on $C$ whose square is the canonical bundle. This definition extends in the 
obvious way to families of spin curves. Let $\mathcal{S}_{2}$ denote the moduli stack of smooth spin curves of genus 2. It is associated to the functor $G: S c h / \mathbb{C} \rightarrow$ Ens defined by

$$
S \mapsto\left\{\begin{array}{l|l}
\text { pairs }\left(\mathcal{C} \rightarrow S, \kappa_{S}\right) & \begin{array}{l}
\mathcal{C} \rightarrow S \text { is a smooth curve of genus } 2 \text { over } S \\
\kappa_{S} \text { is a theta characteristic of } \mathcal{C} \text { over } S
\end{array}
\end{array}\right\}
$$

The moduli stack $\mathcal{S}_{2}$ is a smooth Deligne-Mumford stack which decomposes into two irreducible components $\mathcal{S}_{2}^{+}$and $\mathcal{S}_{2}^{-}$depending on the parity of the theta characteristic. Thus a spin curve $(C, \kappa)$ is in $\mathcal{S}_{2}^{+}$(respectively $\mathcal{S}_{2}^{-}$) if and only if $h^{0}(\kappa)$ is an even (respectively odd) number. Moreover, the forgetful map $\mathcal{S}_{2} \rightarrow \mathcal{M}_{2}$ onto the moduli stack $\mathcal{M}_{2}$ of (smooth) curves of genus 2 is of degree 10 over $\mathcal{S}_{2}^{+}$(respectively 6 over $\mathcal{S}_{2}^{-}$). The functor $G$ admits a coarse moduli space denoted by $S_{2}$. Clearly we have $S_{2}=S_{2}^{+} \sqcup S_{2}^{-}$.

We recall now the compactification $\overline{\mathcal{S}}_{2}$ of $\mathcal{S}_{2}$ constructed by Cornalba in [8] (in fact, the construction works for arbitrary genus, we only need the genus-2 case). A rational component $E \subset X$ of a nodal curve $X$ is called exceptional if $\#(E \cap \overline{X \backslash E})=2$. The curve $X$ is called quasi-stable if $\#(E \cap \overline{X \backslash E}) \geq 2$ for any smooth rational component $E \subset X$ and any two exceptional components are disjoint. A (generalized) spin curve of genus 2 is a triple $(X, \kappa, \beta)$, where $X$ is a quasi-stable curve of (arithmetic) genus 2, $\kappa \in \operatorname{Pic}^{1}(X)$ is a line bundle such that $\kappa_{E}=\mathcal{O}_{E}(1)$ for every exceptional component $E \subset X$, and $\beta: \kappa^{\otimes 2} \rightarrow \omega_{X}$ is a sheaf homomorphism which is generically non-zero along each non-exceptional component of $X$.

When $X$ is smooth, $\kappa$ is an ordinary theta characteristic and $\beta$ is an isomorphism. A family of spin curves over a base scheme $S$ consists of a triple $(\mathcal{X} \stackrel{f}{\rightarrow} S, \eta, \beta)$ where $f: \mathcal{X} \rightarrow S$ is a flat family of quasi-stable curves, $\eta \in \operatorname{Pic}(\mathcal{X})$ is a line bundle and $\beta: \eta^{\otimes 2} \rightarrow \omega_{\mathcal{X}}$ is a sheaf homomorphism, such that at every point $s \in S$ the restriction $\left(\mathcal{X}_{s}, \eta_{s}, \beta_{s}\right)$ is a spin curve of genus 2 . The compactification $\overline{\mathcal{S}}_{2}$ is the stack associated to the functor $G: S \mapsto\{(\mathcal{X} \stackrel{f}{\rightarrow} S, \eta, \beta)\}$. Again one has the obvious decomposition

$$
\overline{\mathcal{S}}_{2}=\overline{\mathcal{S}}_{2}^{+} \sqcup \overline{\mathcal{S}}_{2}^{-}
$$

depending on the parity of $h^{0}(C, \kappa)$.

Let us describe the boundary components of $\bar{S}_{2}^{+}$respectively $\overline{\mathcal{S}}_{2}^{+}$. Denote by $\pi: \bar{S}_{2}^{+} \rightarrow$ $\bar{M}_{2}$ the forgetful map $[X, \kappa, \beta] \mapsto[C]$, where $C$ is the stable model of $X$ is obtained from $X$ by contracting all the exceptional components.

If $[X, \kappa, \beta] \in \pi^{-1}\left(\left[X_{1} \cup_{y} X_{2}\right]\right)$ where $X_{1}$ and $X_{2}$ are elliptic curves intersecting transversally in a point $y$, then necessarily

$$
X:=X_{1} \cup_{y_{1}} E \cup_{y_{2}} X_{2},
$$

where $E$ is an exceptional component such that $X_{1} \cap E=\left\{y_{1}\right\}$ and $X_{2} \cap E=\left\{y_{2}\right\}$ with $\pi\left(y_{i}\right)=y$ for $i=1$ and 2 . Moreover,

$$
\kappa=\left(\kappa_{X_{1}}, \kappa_{X_{2}}, \kappa_{E}=O_{E}(1)\right) \in \operatorname{Pic}^{1}(X),
$$

with theta characteristics $\kappa_{X_{i}}$ on $X_{i}$ for $i=1$ and 2 . The condition $h^{0}(X, \kappa) \equiv 0 \bmod 2$ implies that $\kappa_{X_{1}}$ and $\kappa_{X_{2}}$ have the same parity. We denote

$$
A_{1}:=\text { closure of }\left\{\begin{array}{l|l}
(X, \kappa, \beta) \in \bar{S}_{2}^{+} & \begin{array}{c}
X \text { and } \kappa \text { as above with } h^{0}\left(\kappa_{X_{i}}\right)=0 \text { for } i=1,2 \\
\text { and } \beta=\text { the obvious map (i.e. zero on } E)
\end{array}
\end{array}\right\}
$$


and

$$
B_{1}:=\text { closure of }\left\{(X, \kappa, \beta) \in \bar{S}_{2}^{+} \mid \begin{array}{c}
X \text { and } \kappa \text { as above with } \kappa_{X_{i}}=\mathcal{O}_{X_{i}} \text { for } i=1,2 \\
\text { and } \beta=\text { the obvious map (i.e. zero on } E \text { ) }
\end{array}\right\} .
$$

If $[X, \kappa, \beta] \in \pi^{-1}([C])$ with $C$ an irreducible one-nodal curve, $\nu: \widetilde{C} \rightarrow C$ denotes the normalization of $C$, and

$$
C=\widetilde{C} / y_{1} \sim y_{2}
$$

where $y_{1}$ and $y_{2}$ map to the node $y$ of $C$, then there are two possibilities, namely

$$
X=C \quad \text { or } \quad X=\widetilde{C} \cup_{\left\{y_{1}, y_{2}\right\}} E
$$

with $E$ an exceptional component. In the first case let $\kappa_{\widetilde{C}}:=\nu^{*}(\kappa)$. Then $\kappa_{\widetilde{C}}^{\otimes 2}=K_{\widetilde{C}}\left(y_{1}+\right.$ $\left.y_{2}\right)$ and there is only one choice of gluing the fibres $\kappa_{\widetilde{C}}\left(y_{1}\right)$ and $\kappa_{\widetilde{C}}\left(y_{2}\right)$ to get $\kappa$ such that $h^{0}(X, \kappa) \equiv 0 \bmod 2$. We denote

$$
A_{0}:=\text { closure of }\left\{(X, \kappa, \beta) \in \bar{S}_{2}^{+} \mid X=C, \kappa \text { as above and } \beta \text { the obvious map }\right\} .
$$

If $[X, \kappa, \beta] \in \pi^{-1}([C])$ with $X=\widetilde{C} \cup_{\left\{y_{1}, y_{2}\right\}} E$, then $\kappa_{\widetilde{C}}:=\kappa \otimes \mathcal{O}_{\widetilde{C}}$ is a theta characteristic on $\widetilde{C}$ and $\left.\kappa\right|_{E}=\mathcal{O}_{E}(1)$. Since $H^{0}\left(X, \omega_{X}\right) \simeq H^{0}\left(\widetilde{C}, \omega_{\widetilde{C}}\right)$, it follows that $\kappa_{\widetilde{C}}$ is an even theta characteristic on the elliptic curve $\widetilde{C}$. We denote

$$
B_{0}:=\text { closure of }\left\{\begin{array}{l|l}
(X, \kappa, \beta) \in \bar{S}_{2}^{+} & \begin{array}{c}
X=\widetilde{C} \cup_{\left\{y_{1}, y_{2}\right\}} E, \kappa \text { as above } \\
\text { and } \beta \text { the obvious map }
\end{array}
\end{array}\right\} .
$$

We denote by $\alpha_{0}, \beta_{0}, \alpha_{1}, \beta_{1}$ the corresponding divisors of the stack $\overline{\mathcal{S}}_{2}^{+}$. All in all, we have

$$
\bar{S}_{2}^{+}=S_{2}^{+} \sqcup A_{0} \sqcup B_{0} \sqcup A_{1} \sqcup B_{1} \quad \text { and } \quad \overline{\mathcal{S}}_{2}^{+}=\mathcal{S}_{2}^{+} \sqcup \alpha_{0} \sqcup \beta_{0} \sqcup \alpha_{1} \sqcup \beta_{1} .
$$

\section{The ISOMORPhisM $\mathfrak{S}_{3} \widehat{\mathcal{M}}_{2} \rightarrow \mathcal{S}_{2}^{+}$}

Let $\mathcal{J}_{2}$ be the open substack of the stack of canonically polarized Jacobians of smooth curves of genus 2. In [23] we showed that the Prym map

$$
\operatorname{Pr}: \mathfrak{S}_{3} \widehat{M}_{2} \rightarrow \mathcal{J}_{2}
$$

is a finite surjective morphism of degree 10. The description of the fibres of $\operatorname{Pr}$ hints that there is a relation between the spaces $\mathfrak{S}_{3} \widehat{\mathcal{M}}_{2}$ and $\overline{\mathcal{S}}_{2}^{+}$. Note that the forgetful map $\mathcal{S}_{2}^{+} \rightarrow \mathcal{M}_{2}$ is also of degree 10 . The idea is, to associate to each admissible non-cyclic $\mathfrak{S}_{3}$-covering $[Z \rightarrow X] \in \mathfrak{S}_{3} \widehat{M}_{2}$ the theta divisor of the corresponding Prym variety $P(f)$ of the induced covering $f: Y=Z /\langle\tau\rangle \rightarrow X$, which in this case is a smooth genus 2 curve $C$, together with the theta characteristic arising from a naturally defined 6:1 map $C \rightarrow \mathbb{P}^{1}$.

Theorem 3.1. There is a canonical isomorphism of stacks

$$
\alpha: \mathfrak{S}_{3} \widehat{\mathcal{M}}_{2} \rightarrow \mathcal{S}_{2}^{+} \text {. }
$$


Proof. Let $[h: \mathcal{Z} \rightarrow \mathcal{X}$ over $S] \in \mathfrak{S}_{3} \widehat{\mathcal{M}}_{2}$. So the group $\mathfrak{S}_{3}=\langle\sigma, \tau\rangle$ acts on $\mathcal{Z}$ over $S$. The quotient $\mathcal{Y}:=\mathcal{Z} /\langle\tau\rangle$ is an admissible cover of degree 3 of $\mathcal{X}$ over $S$. Let

$$
f: \mathcal{Y} \rightarrow \mathcal{X}
$$

denote the induced morphism. For any closed point $s \in S$ we associated in [22] and [23] a smooth curve $C(s)$ and a theta characteristic $\kappa(s)$ on $C(s)$ in the following way: Let $\widetilde{f(s)}: \widetilde{\mathcal{Y}(s)} \rightarrow \widetilde{\mathcal{X}(s)}$ denote the normalization of the map $f(s): \mathcal{Y}(s) \rightarrow \mathcal{X}(s)$. The curve $\widetilde{\mathcal{Y}(s)}$ is hyperelliptic of genus 4 (respectively 3 or 2 if $\mathcal{X}(s)$ has 1 or 2 nodes) and there is a commutative diagram (see [22, proof of Theorem 5.1] in the smooth case and [23, proof of Theorem 9.1] in the nodal case)

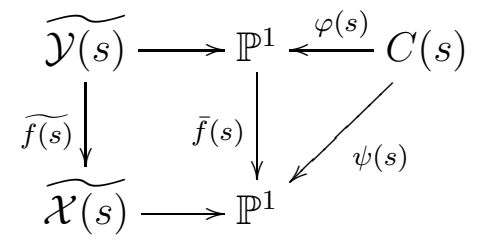

where the horizontal maps of the square are the hyperelliptic coverings (respectively a suitable double cover in the nodal case) and $C(s)$ is a smooth curve of genus 2 , which is a theta divisor of the principal polarization of the Prym variety of the covering $f(s)$, with hyperelliptic cover $\varphi(s)$. To be more precise, in any case $(\mathcal{X}(s)$ smooth or not) there are 6 Weierstrass points $q_{1}, \cdots, q_{6}$ of $\widetilde{\mathcal{Y}(s)}$ and 2 ramification points $p_{1}$ and $p_{2}$ of the double cover $\widetilde{\mathcal{X}(s)} \rightarrow \mathbb{P}^{1}$ such that

$$
f(s)\left(q_{1}\right)=f(s)\left(q_{2}\right)=f(s)\left(q_{3}\right)=p_{1} \quad \text { and } \quad f(s)\left(q_{4}\right)=f(s)\left(q_{5}\right)=f(s)\left(q_{6}\right)=p_{2} .
$$

We can then consider the Prym variety of $f(s)$ as a subvariety of $\operatorname{Pic}^{2}(\widetilde{\mathcal{Y}(s)})$ and the symmetric product $\widetilde{\mathcal{Y}(s)}(2)$ as an open subset of $\operatorname{Pic}^{2} \widetilde{\mathcal{Y}(s)}$ in the usual way. In the smooth case the curve $C(s)$ is given as (see [22, Section 4.5])

$$
C(s)=\left\{\mathcal{O}_{Y}(y+z) \in \operatorname{Pic}^{2}(Y) \mid f(y)=f\left(\iota_{Y} z\right), z \neq y\right\}
$$

with the reduced subscheme structure, where we write $f$ for $f(s)$ and $\iota_{Y}$ denotes the hyperelliptic involution of $Y$. In the nodal case there is a slight modification of this definition for which we refer to [23, Section 8]. With this notation the curve $C(s)$ in any case irreducible and smooth of genus 2. Its Weierstrass points are the 6 points of $C(s)$ :

$$
\omega_{1}=\left[q_{1}+q_{2}\right], \omega_{2}=\left[q_{1}+q_{3}\right], \omega_{3}=\left[q_{2}+q_{3}\right], \omega_{4}=\left[q_{4}+q_{5}\right], \omega_{5}=\left[q_{4}+q_{6}\right], \omega_{6}=\left[q_{5}+q_{6}\right]
$$

(see [22, Proposition 4.18] and [23, Remark 8.9]). The composed map $\psi(s)$ is the morphism given by the pencil generated by the 2 divisors $2 \omega_{1}+2 \omega_{2}+2 \omega_{3}$ and $2 \omega_{4}+2 \omega_{5}+2 \omega_{6}$. Clearly, the linearly equivalent divisors $\omega_{1}+\omega_{2}-\omega_{3}$ and $\omega_{4}+\omega_{5}-\omega_{6}$ of $C(s)$ define an even theta characteristic $\kappa(s)$ on $C(s)$ which is uniquely and canonically determined by the covering $f(s)$.

By construction the pair $(C(s), \kappa(s))$ depends algebraically on $s$. Hence we get a family of pairs

$$
\alpha(h: \mathcal{Z} \rightarrow \mathcal{X}):=(\mathcal{C}, \kappa) \in \mathcal{S}_{2}^{+}
$$


over $S$ with $\mathcal{C}_{s}=C(s)$ and similarly for $\kappa$. Clearly the family is flat as a family of smooth curves.

Conversely, let $[(\mathcal{C}, \kappa)$ over $S] \in \mathcal{S}_{2}^{+}$. So for every closed point $s \in S$, the curve $C(s):=\mathcal{C}_{s}$ is a smooth curve of genus 2 and $\kappa(s):=\kappa_{s}$ an even theta characteristic on it. Recall that if $W=\left\{\omega_{1}, \ldots, \omega_{6}\right\}$ are the Weierstrass points of $C(s)$, the even theta characteristics are of the form $\kappa(s)=\mathcal{O}_{C(s)}\left(\omega_{i}+\omega_{j}-\omega_{k}\right) \simeq \mathcal{O}_{C(s)}\left(\omega_{l}+\omega_{m}-\omega_{n}\right)$ where $\omega_{i}, \omega_{j}, \omega_{k}$ are different points of $W$ and $\omega_{l}, \omega_{m}, \omega_{n}$ their complement in $W$. Hence $\kappa(s)$ determines a partition of $W$ into 2 complemetary subsets of 3 elements of $W$. We use the associated divisors $2 \omega_{i}+2 \omega_{j}+2 \omega_{k}$ and $2 \omega_{l}+2 \omega_{m}+2 \omega_{n}$ to define a degree 6 covering $\psi(s): C(s) \rightarrow \mathbb{P}^{1}$ which certainly factorizes via the hyperelliptic covering $\varphi(s): C(s) \rightarrow \mathbb{P}^{1}$ and a degree 3 covering $\bar{f}(s): \mathbb{P}^{1} \rightarrow \mathbb{P}^{1}$. So we get the right hand triangle of diagram (3.1).

The map $\bar{f}(s)$ is certainly unramified at the 6 points $\bar{\omega}_{i}=\varphi\left(\omega_{i}\right)$ and maps $\bar{\omega}_{i}, \bar{\omega}_{j}$ and $\bar{\omega}_{k}$ to $\bar{p}_{1}$ and $\bar{\omega}_{l}, \bar{\omega}_{m}$ and $\bar{\omega}_{n}$ to $\bar{p}_{2}$ say. According to the Hurwitz formula, $\bar{f}(s)$ is of ramification degree 4 . Suppose first that $\bar{f}(s)$ is simply ramified. Let $\bar{\omega}_{7}, \ldots, \bar{\omega}_{10}$ denote the ramification points and $\bar{p}_{3}, \ldots, \bar{p}_{6}$ the corresponding branch points. Then define hyperelliptic curves $\mathcal{Y}(s)$ with ramification points over $\bar{\omega}_{1}, \ldots, \bar{\omega}_{10}$ and $\mathcal{X}(s)$ with ramification points over $\bar{p}_{1}, \ldots, \bar{p}_{6}$.

Now suppose $\bar{f}(s)$ is simply ramified at $\bar{\omega}_{7}$ and $\bar{\omega}_{8}$ and doubly ramified at $\bar{\omega}_{9}$ and let $\bar{p}_{3}, \bar{p}_{4}$ as well as $\bar{p}_{5}$ the corresponding branch points. In this case, define hyperelliptic curves $\mathcal{Y}(s)$ with ramification points over $\bar{\omega}_{1}, \ldots, \bar{\omega}_{8}$ and a node over $\bar{\omega}_{9}$ and a curve $\mathcal{X}(s)$ of genus 1 with ramification points over $\bar{p}_{1}, \ldots, \bar{p}_{4}$ and a node over $\bar{p}_{5}$.

Finally, suppose $\bar{f}(s)$ is doubly ramified at $\bar{\omega}_{7}$ and $\bar{\omega}_{8}$ and let $\bar{p}_{3}, \bar{p}_{4}$ denote the corresponding branch points. Then define hyperelliptic curves $\mathcal{Y}(s)$ with ramification points over $\bar{\omega}_{1}, \ldots, \bar{\omega}_{6}$ and nodes over $\bar{\omega}_{7}$ and $\bar{\omega}_{8}$ and a curve $\mathcal{X}(s)$ of genus 0 with ramification points over $\bar{p}_{1}$ and $\bar{p}_{2}$ and nodes over $\bar{p}_{3}$ and $\bar{p}_{4}$.

Looking at the ramification one immediately checks that in any case the map $\bar{f}(s)$ lifts to an admissible covering $f(s): \mathcal{Y}(s) \rightarrow \mathcal{X}(s)$. So we obtain a commutative diagram, whose normalization is diagram (3.1). Moreover, it is clear from the constuction that the diagram varies algebraically with $s \in S$. So we get a family $f: \mathcal{Y} \rightarrow \mathcal{X}$ over $S$. Since in any case the coverings $f_{s}=f(s)$ are not Galois, the Galois closure $h: \mathcal{Z} \rightarrow \mathcal{X}$ of $f: \mathcal{Y} \rightarrow \mathcal{X}$ over $S$ is an admissible $\mathfrak{S}_{3}$-cover over $S$. Clearly we obtain an element

$$
\beta((\mathcal{C}, \kappa)):=[h: \mathcal{Z} \rightarrow \mathcal{X} \text { over } S] \in \mathfrak{S}_{3} \widehat{\mathcal{M}}_{2}
$$

over $S$. It is easy to verify that $\beta$ is inverse to $\alpha$.

It remains to check that isomorphisms in the category $\mathfrak{S}_{3} \widehat{\mathcal{M}}_{2}$ are mapped by $\alpha$ to isomorphisms in the category $\mathcal{S}_{2}^{+}$and conversely. This follows from the fact that the maps $\alpha$ and $\beta$ are determined completely by the Weierstrass points and the ramifications of the involved coverings and under isomorphisms these are mapped to Weierstrass points and ramifications of the same type.

As mentioned above, both stacks $\mathfrak{S}_{3} \widehat{\mathcal{M}}_{2}$ and $\mathcal{S}_{2}^{+}$admit coarse moduli spaces $\mathfrak{S}_{3} \widehat{M}_{2}$ and $S_{2}^{+}$. Since an isomorphism of stacks induces an isomorphism of the associated coarse moduli spaces, we obtain as an immediate consequence the following corollary. 
Corollary 3.2. The isomorphism $\alpha: \mathfrak{S}_{3} \widehat{\mathcal{M}}_{2} \rightarrow \mathcal{S}_{2}^{+}$induces an isomorphism of moduli spaces $\mathfrak{S}_{3} \widehat{M}_{2} \rightarrow S_{2}^{+}$, also denoted by $\alpha$.

\section{Non-EXTENSION OF THE CONSTRUCTION DEFINING $\alpha$}

In [23] we considered the following subscheme of the moduli scheme $\mathfrak{S}_{3} \bar{M}_{2}$ of admissible $\mathfrak{S}_{3}$-covers of stable curves of genus 2 :

$$
\mathfrak{S}_{3} \widetilde{M}_{2}:=\left\{\begin{array}{l|l}
{[h: Z \rightarrow X] \in \mathfrak{S}_{3} \bar{M}_{2}} & \begin{array}{l}
p_{a}(Z)=7 \text { and for any node } z \in Z \\
\text { the stabilizer } \operatorname{Stab}(z) \text { is of order 3 }
\end{array}
\end{array}\right\}
$$

and we showed that

$$
\mathfrak{S}_{3} \widetilde{M}_{2}=\mathfrak{S}_{3} \widehat{M}_{2} \sqcup S
$$

where $S$ is the closed subscheme of $\mathfrak{S}_{3} \widetilde{M}_{2}$ consisting of reducible $\mathfrak{S}_{3}$-coverings. In this section we study the question whether the isomorphism $\alpha: \mathfrak{G}_{3} \widehat{M}_{2} \rightarrow S_{2}^{+}$extends to a morphism ${ }_{\mathfrak{S}_{3}} \widetilde{M}_{2} \rightarrow \bar{S}_{2}^{+}$.

Lemma 4.1. There is a non-empty open set $U \subset S$ such that $\alpha$ extends to a holomorphic $\operatorname{map}_{\mathfrak{S}_{3}} \widehat{M}_{2} \sqcup U \rightarrow \bar{S}_{2}^{+}$.

Note that ${ }_{\mathfrak{S}_{3}} \widehat{M}_{2} \sqcup U$ is open in $\widetilde{S}_{3} \widetilde{M}_{2}$ with complement of codimension $\geq 2$.

Proof. The moduli space $\mathfrak{S}_{3} \bar{M}_{2}$ of admissible $G$-covers of stable curves of genus 2 is a normal projective variety (see [2]). So the open subvariety $\mathfrak{S}_{3} \widetilde{M}_{2}$ is also normal. Hence its singular locus is of codimension $\geq 2$. Now $\alpha$ defines a rational map $\bar{\alpha}$ from the smooth variety $\left(\widetilde{S}_{3} \widetilde{M}_{2}\right)_{\text {reg }}$ to $\bar{S}_{2}^{+}$. Since any such rational map extends to a holomorphic map in codimension 1 (see [14, p.491]), this implies the assertion.

However we have,

Proposition 4.2. The construction giving the isomorphism $\alpha: \mathfrak{G}_{3} \widehat{M}_{2} \rightarrow S_{2}^{+}$does not extend to $\mathfrak{S}_{3} \widetilde{M}_{2}$.

In order to see what we have to show, let us recall the definition of the map $\alpha$ in the smooth case. Let $Z \rightarrow X$ be an étale $\mathfrak{S}_{3}$-covering of a smooth curve $X$ of genus 2 and $f: Y \rightarrow X$ a corresponding non-cyclic étale degree-3 covering. Let $h_{X}$ denote the hyperelliptic line bundle on $X$ and $\Theta$ the canonical theta divisor in $\operatorname{Pic}^{3}(Y)$ given by the image of the map $Y^{(3)} \rightarrow \operatorname{Pic}^{3}(Y)$. If $q$ is one of the 2 Weierstrass points of $Y$ such that $f^{-1}(f(q))$ consists of 3 Weierstrass points, we consider the following translate of $\Theta$ :

$$
\Theta_{q}:=\Theta-q \subset \operatorname{Pic}^{2}(Y) .
$$

Let $\mathrm{Nm}: \operatorname{Pic}^{2}(Y) \rightarrow \operatorname{Pic}^{2}(X)$ be the norm map. We define $\widetilde{P}$ as the following translate of the Prym variety $P$ of $f$ :

$$
\widetilde{P}:=\mathrm{Nm}^{-1}\left(h_{X}\right) \subset \operatorname{Pic}^{2}(Y) .
$$

Then we have ([22, Corollary 4.12])

$$
\widetilde{P} \cap \Theta_{q}=\Xi_{1} \cup \Xi_{2} \cup \Xi_{3},
$$


where the $\Xi_{i}$ are algebraically equivalent divisors, each of them defining the principal polarization of $\widetilde{P}$ and thus pairwise isomorphic smooth curves of genus 2 . If $\alpha(f)=(C, \kappa)$, by definition one has

$$
C=\Xi_{1}
$$

For the proof of Proposition 4.2 the theta characteristic $\kappa$ is not relevant.

Now, let $f: Y \rightarrow X$ be the non-cyclic degree-3 covering given by a general element of $S$. So $Y=Y_{1} \cup_{y_{0}} Y_{2}$ and $X=X_{1} \cup_{x_{0}} X_{2}$ with smooth curves $Y_{i}$ of genus 2 (respectively $X_{i}$ of genus 1 ) intersecting transversally in the point $y_{0}$ (respectively $x_{0}$ ) and $f=f_{1} \cup_{y_{0}} f_{2}$ with non-cyclic degree-3 covers $f_{i}: Y_{i} \rightarrow X_{i}$ totally ramified in the point $y_{0 i}\left(=y_{0}\right) \in Y_{i}$ for $i=1,2$ (we denote the point $y_{0}$ by $y_{0 i}$ when considered as a point of $Y_{i}$ ). In order to prove Proposition 4.2, we will show that, defining $\widetilde{P}$ and $\Theta_{q}$ in the same way as in the smooth case, the intersection $\widetilde{P} \cap \Theta_{q}$ is not proper, i.e. not a divisor in $\widetilde{P}$. We need first some preliminaries.

Let $q_{i}^{0}, \ldots, q_{i}^{5}$ denote the Weierstrass points of $Y_{i}$ for $i=1,2$. Then $y_{0}$ is necessarily one of these points, say $y_{0}=q_{1}^{0}=q_{2}^{0}$. For $i=1,2$, the hyperelliptic involution of $X_{i}$ lifts to an involution on $Y_{i}$, which induce an involution on $Y$. Thus we get the following commutative diagram

$$
\begin{gathered}
Y=Y_{1} \cup_{y_{0}} Y_{2} \underset{2: 1}{\stackrel{\gamma=\gamma_{1} \cup_{y_{0}} \gamma_{2}}{\longrightarrow}} \mathbb{P}^{1} \cup_{\gamma\left(y_{0}\right)} \mathbb{P}^{1} \\
f=f_{1} \cup_{x_{0}} f_{2} \downarrow 3: 1 \\
X=X_{1} \cup_{x_{0}} X_{2} \underset{\delta=\delta_{1} \cup_{x_{0}} \delta_{2}}{2: 1} \mathbb{P}^{1} \cup_{\delta\left(x_{0}\right)} \mathbb{P}^{1}
\end{gathered}
$$

where $\gamma_{i}$ and $\delta_{i}$ are the hyperelliptic coverings. It follows that the image of any Weierstrass point under the map $f_{i}$ is a ramification point of $\delta_{i}$. Since $\delta_{i}$ admits 4 ramification points, say $p_{i}^{0}, \ldots, p_{i}^{3}$, we conclude that there is one point $p_{i}^{j}$ such that $f_{i}^{-1}\left(p_{i}^{j}\right)$ consists of 3 Weierstrass points and 3 points $p_{i}^{j}$ such that $f^{-1}\left(p_{i}^{j}\right)$ contains only one Weierstrass point. Without loss of generality we may assume that

$$
f\left(q_{i}^{1}\right)=f\left(q_{i}^{2}\right)=f\left(q_{i}^{3}\right)=p_{i}^{1}
$$

and

$$
f\left(q_{i}^{4}\right)=p_{i}^{2}, \quad f\left(q_{i}^{5}\right)=p_{i}^{3} \quad \text { and } \quad f\left(q_{i}^{0}\right)=p_{i}^{0}=x_{0 i} .
$$

The normalization of $Y$ (respectively $X$ ) is given by $n_{Y}=\iota_{Y_{1}} \cup \iota_{Y_{2}}$ (respectively $\left.n_{X}=\iota_{X_{1}} \cup \iota_{X_{2}}\right)$ where $\iota_{Y_{i}}$ (respectively $\iota_{X_{i}}$ ) denote the canonical embeddings $Y_{i} \rightarrow Y$ (respectively $X_{i} \rightarrow X$ ). They induce canonical isomorphisms of the Picard varieties

$$
n_{Y}^{*}: \operatorname{Pic}(Y) \rightarrow \operatorname{Pic}\left(Y_{1}\right) \times \operatorname{Pic}\left(Y_{2}\right) \quad \text { and } \quad n_{X}^{*}: \operatorname{Pic}(X) \rightarrow \operatorname{Pic}\left(X_{1}\right) \times \operatorname{Pic}\left(X_{2}\right) .
$$

In the sequel we identify both sides, i.e. denote the elements of $\operatorname{Pic}(Y)$ and $\operatorname{Pic}(X)$ by pairs $\left(L_{1}, L_{2}\right)$ with $L_{i} \in \operatorname{Pic}\left(Y_{i}\right)$ (respectively $\left.\operatorname{Pic}\left(X_{i}\right)\right)$.

Now $\operatorname{Pic}^{3}(Y)$ consists of infinitely many components, however there are only 2 components namely $\operatorname{Pic}^{(2,1)}(Y)$ and $\operatorname{Pic}^{(1,2)}(Y)$ (with the obvious notation) which admit a canonical theta divisor (see [5, Proposition 2.2]; these are also the only balanced components in the sense of Caporaso's compactified Picard varieties [8]). Since the situation is 
symmetric in $Y_{1}$ and $Y_{2}$, we work only with $\operatorname{Pic}^{(2,1)}(Y)$. Then the canonical theta divisor is

$$
\Theta:=\left\{L \in \operatorname{Pic}^{(2,1)}(Y)=\operatorname{Pic}^{2}\left(Y_{1}\right) \times \operatorname{Pic}^{1}\left(Y_{2}\right) \mid h^{0}(L) \geq 1\right\}
$$

with reduced subscheme structure. The following lemma is shown by using Riemann-Roch formula.

Lemma 4.3. With the identifications $Y_{1}+y_{01}=\left\{\mathcal{O}_{Y_{1}}\left(y_{1}+y_{01}\right) \mid y_{1} \in Y_{1}\right\}$ and $Y_{2}=$ $\left\{\mathcal{O}_{Y_{2}}\left(y_{2}\right) \mid y_{2} \in Y_{2}\right\}$ we have

$$
\Theta=\left[\left(Y_{1}+y_{01}\right) \times \operatorname{Pic}^{1}\left(Y_{2}\right)\right] \cup\left[\operatorname{Pic}^{2}\left(Y_{1}\right) \times Y_{2}\right] .
$$

If a family of smooth $\mathfrak{S}_{3}$-coverings degenerates to the map $f: Y \rightarrow X$ described above, the Weierstrass point $q$ chosen for the translate of $\Theta$, specializes to one of the points $q_{1}^{i}$ or $q_{2}^{i}$ with $i=1,2$ or 3 . In the latter case $\Theta-q_{2}^{i}$ is a divisor of $\mathrm{Pic}^{(2,0)}$ which is not balanced. So we need to work with one of the $q_{1}^{i}$, say $q=q_{1}^{1}$ and define

$$
\Theta_{q}:=\Theta-q \in \operatorname{Pic}^{(1,1)}(Y) \text {. }
$$

There is exactly one line bundle of degree 2 with $h^{0}=2$ in $\operatorname{Pic}^{(1,1)}(Y)$, respectively $\operatorname{Pic}^{(1,1)}(X)$, namely

$$
h_{Y}=\left(\mathcal{O}_{Y_{1}}\left(q_{01}\right), \mathcal{O}_{Y_{2}}\left(q_{02}\right)\right), \quad \text { respectively } h_{X}=\left(\mathcal{O}_{X_{1}}\left(p_{01}\right), \mathcal{O}_{X_{2}}\left(p_{02}\right)\right) .
$$

If $\operatorname{Nm}_{f}: \operatorname{Pic}^{2}(Y) \rightarrow \operatorname{Pic}^{2}(X)$ denotes the norm map we define, as in the smooth case, $\widetilde{P}$ as the following translate of the Prym variety $P$ of $f$ :

$$
\widetilde{P}:=\operatorname{Nm}_{f}^{-1}\left(h_{X}\right) \subset \operatorname{Pic}^{(1,1)}(Y) .
$$

Lemma 4.4. The intersection $\Theta_{q} \cap \widetilde{P}$ contains the 3 pairwise disjoint curves

$$
\Sigma_{i}:=\left\{\left(\mathcal{O}_{Y_{1}}\left(q_{1}^{i}+y_{01}-q\right), \mathcal{O}_{Y_{2}}\left(y_{02}+y_{2}-y_{2}^{\prime}\right)\right) \mid y_{2} \in Y_{2}, y_{2}^{\prime} \in f_{2}^{-1} f_{2}\left(y_{2}\right)\right\}
$$

for $i=1,2,3$ which are $3: 1$-coverings of $Y_{2}$.

Proof. According to Lemma 4.3 we have $\Sigma_{i} \subset \Theta_{q}$. By the identifications (4.2), $\mathrm{Nm}_{f}=$ $\mathrm{Nm}_{f_{1}} \times \mathrm{Nm}_{f_{2}}$ and one computes

$$
\begin{aligned}
\operatorname{Nm}_{f}\left(\left(\mathcal { O } _ { Y _ { 1 } } \left(q_{1}^{i}+\right.\right.\right. & \left.y_{01}-q\right), \mathcal{O}_{Y_{2}}\left(y_{02}+y_{2}-\iota_{Y_{2}}\left(y_{2}\right)\right)= \\
= & \left(\mathcal{O}_{X_{1}}\left(p_{1}+x_{01}-p_{1}\right), \mathcal{O}_{X_{2}}\left(x_{02}+f_{2}\left(y_{2}\right)-f_{2}\left(y_{2}\right)\right)\right. \\
& =\left(\mathcal{O}_{X_{1}}\left(x_{01}\right), \mathcal{O}_{X_{2}}\left(x_{02}\right)\right)=h_{X} .
\end{aligned}
$$

So $\Sigma_{i} \subset \widetilde{P}$. The curves $\Sigma_{i}$ are pairwise disjoint, since $q=q_{1}^{1}$ and the line bundles $\mathcal{O}_{X_{1}}\left(q_{1}^{i}+y_{01}-q_{1}^{1}\right)$ are pairwise different. The last assertion follows from the fact that $f_{2}$ is a 3 : 1 -covering and hence there are 3 preimages $y_{2}^{\prime} \in f_{2}^{-1} f_{2}\left(y_{2}\right)$.

Proof of Proposition 4.2. We may consider $\operatorname{Pic}^{(1,1)}(Y)$ as an abelian variety and $\widetilde{P}$ as an abelian subvariety, since both contain the distinguished point $h_{Y}$. According to [23, Proposition 5.3],

$$
\widetilde{P}=P_{1} \times P_{2}
$$

with elliptic curves $P_{i}=\operatorname{Prym}\left(f_{i}\right)$ for $i=1,2$ and canonical principal polarization. On the other hand, the principal polarization of $\operatorname{Pic}^{(1,1)}(Y)$ is defined by the divisor $\Theta_{q}$. So if the construction of $\alpha$ would extend to the closed subvariety $S$, the divisor $\Theta_{q}$ would 
restrict to a divisor defining the threefold of the canonical principal polarization of $\widetilde{P}$. Being pairwise disjoint, the curves $\Sigma_{i}$ would define the canonical principal polarization i.e. would be isomorphic to $P_{1} \times\{0\} \cup\{0\} \times P_{2}$. But this contradicts Lemma 4.4 since, the curve $Y_{2}$ being of genus 2, any 3:1 covering is of arithmetic genus $>2$ and hence $\Sigma_{i}$ cannot define a principal polarization.

\section{The NeF CONE OF $\bar{S}_{2}^{+}$}

In this section we compute the cone of numerically effective divisors of $\bar{S}_{2}^{+}$in the rational Picard group Pic $\mathbb{Q}_{\mathbb{Q}}\left(\bar{S}_{2}^{+}\right)$. For this we consider the moduli space $\bar{M}_{0,[3,3]}$ of stable curves of genus 0 with 6 unordered marked points partitioned into 2 sets of 3 points. If $\bar{M}_{0,6}$ denotes the usual moduli space of stable 6-pointed curves of genus 2 and

$$
G:=\left(\mathfrak{S}_{3} \times \mathfrak{S}_{3}\right) \rtimes\langle\tau\rangle
$$

where the first $\mathfrak{S}_{3}$ acts on the numbers $1,2,3$, the second on the numbers 4,5,6 and $\tau=(14)(25)(36)$, then

$$
\bar{M}_{0,[3,3]}=\bar{M}_{0,6} / G \text {. }
$$

Here we consider the numbers as indices of the marked points. We use the fact (see [20, Lemma 20]) that there is a canonical isomorphism

$$
\bar{M}_{0,[3,3]} \simeq \bar{S}_{2}^{+} .
$$

In the sequel we often identify both spaces and denote corresponding divisors by the same letter. Moreover, the composed map $\bar{M}_{0,6} \rightarrow \bar{S}_{2}^{+}$maps the boundary of $\bar{M}_{0,6}$ onto the boundary of $\bar{S}_{2}^{+}([20])$.

Recall that the boundary of $\bar{M}_{0,6}$ consists of divisors $\Delta_{S}$, where $S \subset\{1, \ldots, 6\}$ with $|S|,\left|S^{c}\right| \geq 2$. Each $\Delta_{S}$ is the closure of the points corresponding to reducible curves with one node and where $S$ points are marked in one component. We denote the class of $\Delta_{S}$ in $\operatorname{Pic}_{\mathbb{Q}}\left(\bar{M}_{0,6}\right)$ by the same letter.

The divisors in $\bar{M}_{0,[3,3]}$ can be regarded as the divisors in $\bar{M}_{0,6}$ which are invariant under the action of $G$. The isomorphism $\bar{M}_{0,[3,3]} \simeq \bar{S}_{2}^{+}$is defined in a natural way by associating to a rational curve with six marked points the admissible double covering ramified over those points $([20])$. Moreover, it maps the following $G$-invariant divisors into the boundary divisors of $\bar{S}_{2}^{+}$(see [20, table in section 3.1]) :

$$
\begin{aligned}
\Delta^{11} & :=\sum_{S \in O r b_{G}(12)} \Delta_{S} \\
\Delta^{12} & :=\sum_{S \in O r b_{G}(14)} \Delta_{S} \\
\Delta_{123}^{c} & \mapsto A_{0} \\
\sum_{S \in O r b_{G}(124)} \Delta_{S} & \mapsto A_{1} \\
\Delta_{123}:=\Delta_{\{1,2,3\}} & \mapsto B_{1}
\end{aligned}
$$

In order to see the $G$-invariance of these divisors, use the fact that if $\left\{i_{1}, \ldots, i_{6}\right\}=$ $\{1, \ldots, 6\}$, then $\Delta_{\left\{i_{1}, i_{2}, i_{3}\right\}}=\Delta_{\left\{i_{4}, i_{5}, i_{6}\right\}}$. When we consider the $G$-invariant classes $\Delta^{11}, \Delta^{12}$, 
$\Delta_{123}^{c}$ and $\Delta_{123}$ as elements of $\operatorname{Pic}_{\mathbb{Q}}\left(\bar{M}_{0,[3,3]}\right)$, we denote them respectively by $A_{0}, B_{0}, A_{1}$ and $B_{1}$. According to [20] the classes $A_{0}, B_{0}, A_{1}$ and $B_{1}$ generate the $\mathbb{Q}$-vector space $\operatorname{Pic}_{\mathbb{Q}}\left(\bar{M}_{0,[3,3]}\right)$.

In order to compute the nef cone of $\bar{M}_{0,[3,3]}$ we introduce some $F$-curves. An $F$-curve in $\bar{M}_{0,6}$ is obtained as the image of the map $\nu: \bar{M}_{0,4} \rightarrow \bar{M}_{0,6}$ defined by attaching one 3pointed curve at one of the 4 marked points or two 2-pointed curves at two of the marked points ([13, end of Section 2]). Note that, as a complete intersection curve in $\bar{M}_{0,6}$, the curve $F$ always consists of 3 irreducible components, in the first case the attached curve is semistable with 2 irreducible components, whereas in the the second case the 2 attached curves are irreducible.

We consider the following $F$-curves. Let $\Gamma_{1}$ (respectively $\Gamma_{2}$ ) denote the curve in $\bar{M}_{0,6}$ whose elements are constructed by attaching to a spine labelled with the subset $\{1,2,3\}$ (respectively $\{1,2,4\}$ ) of $\{1, \ldots, 6\}$ to a 4 th point a rational curve consisting of 2 components where the component directly attached to the spine is labelled with 4 (respectively 3 ) and the other curve with 5 and 6 (see Figure 1 ). This defines a curve in $\bar{M}_{0,6}$, since the 4th point of the spine moves freely, whereas the other components have 3 marked points: the nodes and the labelled points.

Let $\Gamma_{3}$ be the curve constructed by attaching two 2-pointed irreducible curves to the spine where exactly one of the attaching points moves and the 2 points on the spine are labelled with $\{1,2\}$. Similarly, we define $\Gamma_{4}$ (respectively $\Gamma_{5}$ ) by attaching two 2 -pointed curves and where the labelled points on the spine are $\{1,4\}$ and one tail is labelled with $\{2,3\}$ (respectively $\{2,5\}$ ) (see Figure 1 ).
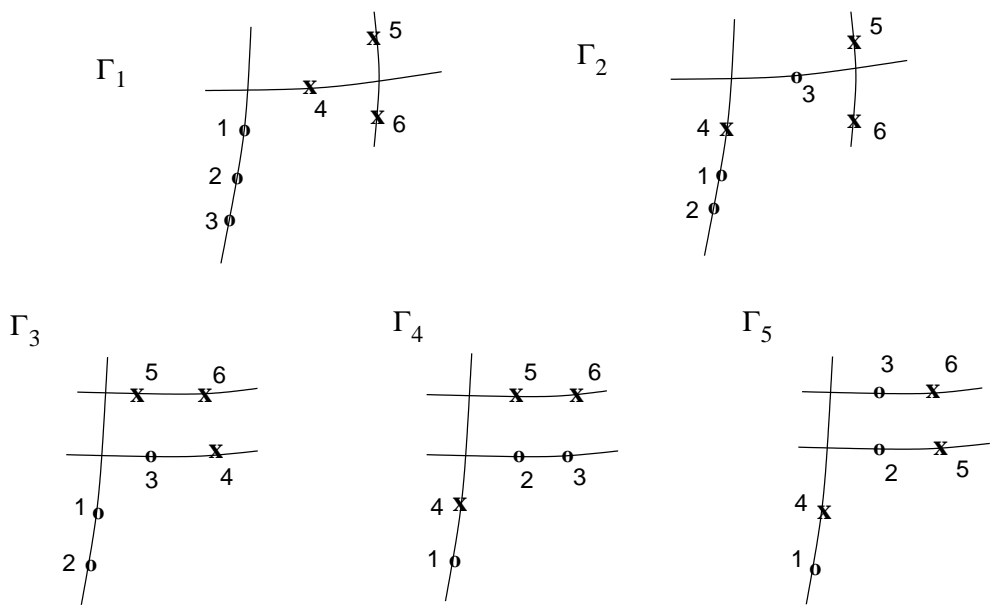

Figure 1. Generic points of the F-curves

In principle one should consider the orbit of an $F$-curve under the action of the group $G$, but in order to determine the inequalities defining the nef-cone it will be sufficient to intersect the divisors with a representative of the orbit.

Lemma 5.1. A rational divisor class $a A_{0}+b B_{0}+c A_{1}+d B_{1}$ of $\bar{S}_{2}^{+}$is nef (respectively ample) if and only if the corresponding class $D:=a \Delta^{11}+b \Delta^{12}+c \Delta_{123}^{c}+d \Delta_{123}$ of $\bar{M}_{0,6}$ 
satisfies

$$
\left(D \cdot \Gamma_{i}\right) \geq 0 \quad(\text { respectively }>0)
$$

for $i=1, \ldots, 5$.

Proof. The class of $D$ being $G$-invariant, $D$ is nef as a divisor of $\bar{M}_{0,[3,3]}$ if and only if it is nef as a divisor of $\bar{M}_{0,6}$. Hence according to [18, Theorem 1.3] $D$ is nef if and only if it intersects any $F$-curve of $\bar{M}_{0,6}$ non-negatively. Since $D$ is $G$-invariant, it suffices to check this for a representative of the $G$-orbits of $F$-curves. Now it is easy to check that there are exactly $6 G$-orbits with representatives $\Gamma_{1}, \ldots, \Gamma_{5}$ and $\Gamma_{2}^{\prime}$. Here $\Gamma_{2}^{\prime}$ differs from $\Gamma_{2}$ only by labelling the middle component with 5 (instead of 3) and the last component with 3 and 6 (instead of 5 and 6). However, by [18, Lemma 4.3] $\Gamma_{2}^{\prime}$ is numerically equivalent to $\Gamma_{2}$. So this implies the assertion on the nefness and also on the ampleness of the divisor class.

Lemma 5.2. The intersection numbers of the divisors $\Delta^{11}, \ldots, \Delta_{123}$ with $\Gamma_{i}$ are

$$
\begin{array}{llll}
\left(\Delta^{11} \cdot \Gamma_{1}\right)=3, & \left(\Delta^{12} \cdot \Gamma_{1}\right)=0, & \left(\Delta_{123}^{c} \cdot \Gamma_{1}\right)=0, & \left(\Delta_{123} \cdot \Gamma_{1}\right)=-1 ; \\
\left(\Delta^{11} \cdot \Gamma_{2}\right)=1, & \left(\Delta^{12} \cdot \Gamma_{2}\right)=2, & \left(\Delta_{123}^{c} \cdot \Gamma_{2}\right)=-1, & \left(\Delta_{123} \cdot \Gamma_{2}\right)=0 ; \\
\left(\Delta^{11} \cdot \Gamma_{3}\right)=0, & \left(\Delta^{12} \cdot \Gamma_{3}\right)=-1, & \left(\Delta_{123}^{c} \cdot \Gamma_{3}\right)=2, & \left(\Delta_{123} \cdot \Gamma_{3}\right)=0 ; \\
\left(\Delta^{11} \cdot \Gamma_{4}\right)=-2, & \left(\Delta^{12} \cdot \Gamma_{4}\right)=1, & \left(\Delta_{123}^{c} \cdot \Gamma_{4}\right)=1, & \left(\Delta_{123} \cdot \Gamma_{4}\right)=1 ; \\
\left(\Delta^{11} \cdot \Gamma_{5}\right)=0, & \left(\Delta^{12} \cdot \Gamma_{5}\right)=-1, & \left(\Delta_{123}^{c} \cdot \Gamma_{5}\right)=2, & \left(\Delta_{123} \cdot \Gamma_{5}\right)=0 ;
\end{array}
$$

Proof. This follows by an immediate computation using [18, Lemma 4.3] which gives the intersection numbers of any boundary divisor with any $F$-curve of $\bar{M}_{0, n}$.

From this we immediately conclude the following criterion for a rational divisor class on $\bar{M}_{0,[3,3]}=\bar{S}_{2}^{+}$to be nef or ample.

Proposition 5.3. $A \mathbb{Q}$-divisor $D \equiv a A_{0}++b B_{0}+c A_{1}+d B_{1}$ is nef (respectively ample) if and only if the following inequalities are satisfied

$$
3 a \geq d, \quad a+2 b \geq c, \quad 2 c \geq b \quad \text { and } \quad b+c+d \geq 2 a
$$

(respectively all inequalities are strict).

Proof. According to Lemma 5.2 and the identifications (5.1), ..., (5.4) we have

$$
\begin{gathered}
\left(D \cdot \Gamma_{1}\right)=3 a-d, \quad\left(D \cdot \Gamma_{2}\right)=a+2 b-c, \\
\left(D \cdot \Gamma_{3}\right)=\left(\Gamma \cdot \Gamma_{5}\right)=-b+2 c, \quad\left(D \cdot \Gamma_{4}\right)=-2 a+b+c+d .
\end{gathered}
$$

So Lemma 5.1 implies the assertion.

Remark 5.4. According to [20] the vector space $\operatorname{Pic}_{\mathbb{Q}}\left(\bar{S}_{2}^{+}\right)=\operatorname{Pic}_{\mathbb{Q}}\left(\bar{M}_{0,[3,3]}\right)$ is of dimension 3 . Hence there is a non-trivial relation

$$
a A_{0}+b B_{0}+c A_{1}+d B_{1}=0
$$

between the classes $A_{i}$ and $B_{i}$. Intersecting with the curves $\Gamma_{i}$ we get the system of equations

$$
3 a=d, \quad a+2 b=c, \quad 2 c=b, \quad b+c+d=2 a .
$$


This gives the relation

$$
3 A_{0}-2 B_{0}-A_{1}+9 B_{1}=0 .
$$

in $\operatorname{Pic}_{\mathbb{Q}}\left(\bar{S}_{2}^{+}\right)$. Note that this is different from the relation proved in [20, Lemma 21 (ii)].

Since $\operatorname{Pic}_{\mathbb{Q}}\left(\bar{S}_{2}^{+}\right)$is of dimension 3, (5.5) implies that $\left\{A_{0}, B_{0}, B_{1}\right\}$ is a basis of $\operatorname{Pic}_{\mathbb{Q}}\left(\bar{S}_{2}^{+}\right)$. In terms of this basis Proposition 5.3 can be rephrased as

Corollary 5.5. A divisor $D=a A_{0}+b B_{0}+c B_{1}$ of $\bar{S}_{2}^{+}$is nef (respectively ample) if and only if

$$
b \leq 0 \quad \text { and } \quad \max \left(\frac{1}{3} c,-2 b\right) \leq a \leq \frac{1}{2}(b+c)
$$

\section{Proof of part (1) of Theorem 2}

As in Section 2 let $\overline{\mathcal{S}}_{2}^{+}$and $\overline{\mathcal{M}}_{2}$ denote the corresponding moduli stacks of $\bar{S}_{2}^{+}$and $\bar{M}_{2}$. Consider the following commutating diagram:

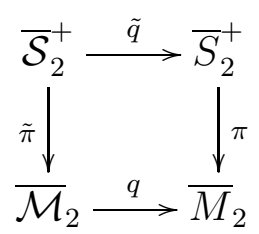

where $\pi$ (resp. $\tilde{\pi}$ ) is the forgetful map $[(C, \kappa)] \mapsto[C]$ and $\tilde{q}$ and $q$ are the natural maps. The first aim is to give a formula for the class $K_{\mathcal{S}_{2}^{+}}$in terms of divisors in $\bar{S}_{2}^{+}$.

Let $E \subset M_{2}$ the locus of the bielliptic curves. Any bielliptic curve $C \in E$ has an equation of the form

$$
y^{2}=\left(x^{2}-x_{1}^{2}\right)\left(x^{2}-x_{2}^{2}\right)\left(x^{2}-x_{3}^{2}\right)
$$

Thus the Weierstrass points of $C$ are $\pm x_{1}, \pm x_{2}, \pm x_{3}$. Let $\rho$ denote the bielliptic involution in $C$.

Lemma 6.1. The preimage of the bielliptic locus $E$ in $\bar{S}_{2}^{+}$decomposes as

$$
\pi^{*}(E)=\widetilde{E} \cup \widetilde{E}^{\prime}
$$

where $\widetilde{E}$ represents the spin curves $(C, \kappa)$ with $C$ admitting a bielliptic involution $\rho$ such that $\rho^{*}(\kappa)=\kappa$. Moreover the map $\pi: \widetilde{E} \mapsto E$ is finite of degree 4 . In particular $\widetilde{E}$ is the bielliptic locus of $\bar{S}_{2}^{+}$.

Proof. Given a bielliptic curve $[C] \in E$ with equation (6.1) and Weierstrass points $\pm x_{1}, \pm x_{2}, \pm x_{3}$, it suffices to check that there are exactly 4 even theta characteristics on $C$ which are invariant under the action of $\rho$.

We set $x_{i}^{\prime}:=-x_{i}$ for $i=1,2,3$. The involution $\rho$ acts on the Weierstrass points by $x_{i} \mapsto x_{i}^{\prime}$. The even theta characteristics on $C$ are of the form

$$
p_{1}+p_{2}-p_{3} \sim p_{4}+p_{5}-p_{6}
$$

where $\left\{p_{1}, \ldots, p_{6}\right\}$ is the set of Weierstrass points. Using this equivalence is easy to verify that exactly the following theta characteristics are fixed by $\rho$ :

$$
x_{1}+x_{2}-x_{3}, \quad x_{1}+x_{2}-x_{3}^{\prime}, \quad x_{1}+x_{2}^{\prime}-x_{3} \quad x_{1}^{\prime}+x_{2}-x_{3} .
$$


Thus the action of $\rho$ decomposes $\pi^{*} E$ into to divisors: $\widetilde{E}$ where $\rho$ fixes the theta characteristics and $\widetilde{E}^{\prime}$ where $\rho$ permutes non-trivially the remaining 6 theta characteristics, which are

$$
x_{i}+x_{i}^{\prime}-x_{j}, \quad 1 \leq i, j \leq 3, i \neq j .
$$

Recall that $\bar{M}_{2} \backslash M_{2}=\Delta_{0} \cup \Delta_{1}$, where $\Delta_{0}$ (respectively $\Delta_{1}$ ) is the closure of the locus of irreducible nodal curves (respectively of the reducible curves) of genus 2 .

Lemma 6.2. The forgetful map $\pi: \bar{S}_{2}^{+} \rightarrow \bar{M}_{2}$ is simply ramified on $B_{0}$ and unramified everywhere else in codimension 1.

Proof. Clearly $\pi$ is étale of degree 10 over the smooth locus of $M_{2}$. We have $\pi^{-1}\left(\Delta_{0}\right)=$ $A_{0} \cup B_{0}$. From the construction of the divisor $A_{0}$ we obtain that $\operatorname{deg}\left(\left.\pi\right|_{A_{0}}\right)=4$ (since an elliptic curve admits exactly 4 theta characteristics). Over a semistable curve $\tilde{C} \cup_{y_{1}, y_{2}} R$ with $g(\tilde{C})=1$ and $R$ a rational component there are exactly 3 even theta characteristics (since an elliptic curve admits exactly 3 even theta characteristics). From $\operatorname{deg}\left(\left.\pi\right|_{B_{0}}\right)=6$ we deduce that $\pi$ is simply ramified in $B_{0}$. One verifies that $\pi$ is étale over $\Delta_{1}$ since $\pi^{-1}\left(\Delta_{1}\right)=A_{1} \cup B_{1}, \operatorname{deg}\left(\left.\pi\right|_{A_{1}}\right)=9$ and $\pi$ maps $B_{1}$ isomorphically onto $\Delta_{1}$.

We use the previous lemmas to compute the class of $K_{\overline{\mathcal{S}}_{2}^{+}}$.

\section{Lemma 6.3.}

$$
K_{\overline{\mathcal{S}}_{2}^{+}}=\tilde{q}^{*}\left(K_{\bar{S}_{2}^{+}}+\frac{1}{2}\left(A_{1}+B_{1}\right)+\frac{1}{2} \widetilde{E}\right) .
$$

where $\widetilde{E}$ denotes the spin bielliptic locus of $\bar{S}_{2}^{+}$.

Proof. The map $\tilde{q}$ is ramified along the locus of points in $\bar{S}_{2}^{+}$which admits an automorphism group bigger than $\langle\iota\rangle$ with $\iota$ the hyperelliptic involution. It suffices to compute the codimension-one components of this locus. In [16] the locus of such curves have been computed for $\bar{M}_{2}$. It consists of the locus of bielliptic curves plus the boundary divisor $\Delta_{1}$. One immediately checks that the automorphism group of a general spin curve in $\pi^{-1}\left(\Delta_{1}\right)=A_{1} \cup B_{1}$ is abelian of order 4 . This together with the Lemma 6.1 proves the lemma.

From the proof we also get that the boundary class $\delta$ of the stack $\overline{\mathcal{S}}_{2}^{+}$is given by

$$
\delta=\tilde{q}^{*}\left(A_{0}+B_{0}+\frac{1}{2} A_{1}+\frac{1}{2} B_{1}\right) .
$$

So we get as an immediate consequence

Corollary 6.4. (Ramification formula for $\tilde{q}$ ): For every $\epsilon \in \mathbb{Q}$,

$$
K_{\overline{\mathcal{S}}_{2}^{+}}+\epsilon \delta=\tilde{q}^{*}\left(K_{\bar{S}_{2}^{+}}+\epsilon\left(A_{0}+B_{0}\right)+\frac{\epsilon+1}{2}\left(A_{1}+B_{1}\right)+\frac{1}{2} \widetilde{E}\right) .
$$

Proposition 6.5. The divisor $K_{\overline{\mathcal{S}}_{2}^{+}}+\epsilon \delta$ is nef (respectively ample) if and only if $\epsilon \geq \frac{57}{25}$ (respectively the inequality is strict). 
Proof. From [16, Section 4.2 and Proposition 3.6] we have

$$
K_{\bar{M}_{2}} \equiv-\frac{11}{5} \Delta_{0}-\frac{16}{5} \Delta_{1}, \quad E \equiv 3 \Delta_{0}+12 \Delta_{1} .
$$

Since by Lemma 6.2 we have $\pi^{*} \Delta_{0}=A_{0}+2 B_{0}$ and $\pi^{*} \Delta_{1}=A_{1}+B_{1}$, we get

$$
\begin{aligned}
K_{\overline{\mathcal{S}}_{2}^{+}} & =\pi^{*}\left(-\frac{11}{5} \Delta_{0}-\frac{16}{5} \Delta_{1}\right)+B_{0} \\
& =-\frac{11}{5} A_{0}-\frac{17}{5} B_{0}-\frac{16}{5} A_{1}-\frac{16}{5} B_{1} .
\end{aligned}
$$

Moreover by Lemma 6.1 and the fact that $\pi$ is étale of degree 10 over $M_{2}$ we get

$$
\begin{aligned}
\widetilde{E} & =\frac{4}{10} \pi^{*}(E) \\
& =\frac{4}{10}\left(\pi^{*}\left(3 \Delta_{0}+12 \Delta_{1}\right)\right)=\frac{6}{5} A_{0}+\frac{12}{5} B_{0}+\frac{24}{5} A_{1}+\frac{24}{5} B_{1}
\end{aligned}
$$

So Corollary 6.4 implies $K \overline{\mathcal{S}_{2}^{+}}+\epsilon \delta=\tilde{q}^{*} F$ with

$$
F=\left(\epsilon-\frac{8}{5}\right) A_{0}+\left(\epsilon-\frac{11}{5}\right) B_{0}+\left(\frac{\epsilon}{2}-\frac{3}{10}\right) A_{1}+\left(\frac{\epsilon}{2}-\frac{3}{10}\right) B_{1} .
$$

The inequalities of Proposition 5.3 applied to $F$ give the following conditions for the class $F$ to be ample:

$$
\epsilon>\frac{9}{5}, \quad \epsilon>\frac{57}{25}
$$

(the last two inequalities do not impose conditions on $\epsilon$ ).

Let us recall the definition of a $\log$ canonical model. Let $X$ be a normal projective variety and $D=\sum_{i=1}^{n} a_{i} D_{i}$ a $\mathbb{Q}$-divisor such that $K_{X}+D$ is $\mathbb{Q}$-Cartier and $0 \leq a_{i} \leq 1$.

Definition 6.6. The pair $(X, D)$ is a strict log canonical model if $K_{X}+D$ is ample, $(X, D)$ has $\log$ canonical singularities, and $X \backslash \cup_{i} D_{i}$ has canonical singularities.

We will also need the following proposition, whose proof we refer to [19, 20.2, 20.3]):

Proposition 6.7. Let $Y$ be a smooth variety and $f: Y \rightarrow X$ be a finite dominant morphism to a normal variety $X$. Let $D=\sum_{i=1} a_{i} D_{i}, 0 \leq a_{i} \leq 1$ be a $\mathbb{Q}$-divisor containing all the divisorial components of the branch locus of $f$. Let $\bar{D}$ be a $\mathbb{Q}$-divisor on $Y$ such that $\operatorname{supp}\left(f^{-1}(D)\right)=\operatorname{supp}(\bar{D})$ and $f^{*}\left(K_{X}+D\right)=K_{Y}+\bar{D}$. Then $(X, D)$ has log canonical singularities along $D$ if and only if $(Y, \bar{D})$ has log canonical singularities along $\bar{D}$.

Proof of Theorem 2(1). Proposition 6.5 shows the required ampleness of the divisor F. In order to verify the singularity conditions of $\bar{S}_{2}^{+}$we will use the results in [16, Theorem 4.10]. Consider the finite map $\pi: \bar{S}_{2}^{+} \rightarrow \bar{M}_{2}$. We have that $\bar{M}_{2}$ has canonical singularities away from $\Delta_{0}$ and $\Delta_{1}([16])$. Since $\pi_{S_{2}^{+}}$is étale, it follows that $\bar{S}_{2}^{+}$has canonical singularities away from $\pi^{-1}\left(\Delta_{0} \cup \Delta_{1}\right)=A_{0} \cup B_{0} \cup A_{1} \cup B_{1}$. In particular it has canonical singularities away from the boundary divisors $A_{0}, B_{0}, A_{1}, B_{1}$ and $\widetilde{E}$. 
Since $\bar{M}_{2}$ has $\log$ canonical singularities along $\Delta_{0}, \Delta_{1}$ and $E$ ([16]), by Proposition $6.7 \bar{S}_{2}^{+}$has $\log$ canonical singularities along $\pi^{-1}\left(\Delta_{0} \cup \Delta_{1} \cup E\right)$ and hence also along $A_{0} \cup B_{0} \cup A_{1} \cup B_{1} \cup \widetilde{E}$, since $\bar{M}_{2}$ is smooth at $E$ and $\pi$ is étale over $E$.

\section{The invariant-THEORETICAL COMPACTIFICATION OF $S_{2}^{+}$}

Let $C$ be a smooth projective curve of genus 2 over $k$. Let $\mathbb{F}_{2}^{4}$ denote the 4 -dimensional vector space over $\mathbb{F}_{2}$ equipped with a fixed symplectic form. The group of 2-division points $J C[2]$ of the Jacobian of $C$ is isomorphic to $\mathbb{F}_{2}^{4}$ and admits a canonical symplectic form, the Weil form. A curve of genus 2 with a level-2 structure consists of a pair $(C, \varphi)$ with a curve $C$ of genus 2 and a level-2 structure $\varphi$, that is a symplectic isomorphism $\varphi: \mathbb{F}_{2}^{4} \rightarrow J C[2]$. Let $M_{2}(2)$ denote the coarse moduli space of such pairs. It can be constructed as follows:

The hyperelliptic covering $C \rightarrow \mathbb{P}^{1}$ is ramified exactly in the 6 Weierstrass points $p_{1}, \ldots, p_{6}$ with images $x_{1}, \ldots x_{6} \in \mathbb{P}^{1}$. It is well-known (see [11]) that a level-2 structure on $C$ is equivalent to an order of the set of Weierstrass points of $C$. Denote by

$$
\Delta_{6}=\left\{\left(x_{1}, \ldots, x_{6}\right) \in\left(\mathbb{P}^{1}\right)^{6} \mid x_{i}=x_{j} \text { for some } i \neq j\right\}
$$

the diagonal of the sixfold cartesian product $\left(\mathbb{P}^{1}\right)^{6}$ of $\mathbb{P}^{1}$. Then

$$
M_{2}(2) \simeq\left[\left(\mathbb{P}^{1}\right)^{6} \backslash \Delta_{6}\right] / / \mathrm{SL}_{2}(k)
$$

where $\mathrm{SL}_{2}(k)$ acts in the usual way on $\mathbb{P}^{1}$ and diagonally on $\left(\mathbb{P}^{1}\right)^{6}$. The forgetful map

$$
M_{2}(2) \rightarrow M_{2}, \quad(C, \varphi) \mapsto C
$$

onto the coarse moduli space $M_{2}$ of smooth curves of genus 2 corresponds to the quotient map

$$
\left[\left(\mathbb{P}^{1}\right)^{6} \backslash \Delta_{6}\right] / / \mathrm{SL}_{2}(k) \rightarrow\left\{\left[\left(\mathbb{P}^{1}\right)^{6} \backslash \Delta_{6}\right] / / \mathrm{SL}_{2}(k)\right\} / \mathfrak{S}_{6}
$$

where the action of the symmetric group $\mathfrak{S}_{6}$ is induced by its permutation action on $\left(\mathbb{P}^{1}\right)^{6}$.

Recall from [24 that an even theta characteristic on $C$ is the line bundle given by a divisor $p_{i_{1}}+p_{i_{2}}-p_{i_{3}}$ where the $p_{i_{j}}$ are different Weierstrass points and $p_{j_{1}}+p_{j_{2}}-$ $p_{j_{3}}$ defines the same theta characteristic if and only if either $\left\{j_{1}, j_{2}, j_{3}\right\}=\left\{i_{1}, i_{2}, i_{3}\right\}$ or $\left\{i_{1}, i_{2}, i_{3}, j_{1}, j_{2}, j_{3}\right\}=\{1, \ldots, 6\}$. This implies that the even theta characteristics of $C$ are in a natural bijective correspondence with the 3 -element subsets of the set $\{1, \ldots, 6\}$ modulo the involution $\left\{i_{1}, i_{2}, i_{3}\right\} \mapsto\{1, \ldots, 6\} \backslash\left\{i_{1}, i_{2}, i_{3}\right\}$. In order to construct the coarse moduli space $S_{2}^{+}$of even spin curves of genus 2 consider again the subgroup

$$
G:=\left(\mathfrak{S}_{3} \times \mathfrak{S}_{3}\right) \rtimes\langle\tau\rangle
$$

of $\mathfrak{S}_{6}$ as defined at the beginning of Section 5 .

Clearly the stabilizer of an even theta characteristic given by $\left\{i_{1}, i_{2}, i_{3}\right\}$ is conjugate to $G$. Hence we obtain an isomorphism

$$
S_{2}^{+} \stackrel{\simeq}{\longrightarrow} M_{2}(2) / G=\left\{\left[\left(\mathbb{P}^{1}\right)^{6} \backslash \Delta_{6}\right] / / \mathrm{SL}_{2}(k)\right\} / G .
$$

In [11, p.17] it is shown that a natural compactification of $M_{2}(2)$, the invariant-theoretical compactification, which we denote by ${\overline{M_{2}(2)}}^{i n v}$, is isomorphic to the Segre cubic threefold. 
In $\mathbb{P}^{5}=\mathbb{P}^{5}\left(t_{0}, \ldots, t_{5}\right)$ the Segre cubic is given by the equations

$$
s_{1}:=\sum_{i=0}^{5} t_{i}=0 \quad \text { and } \quad s_{3}:=\sum_{i=0}^{5} t_{i}^{3}=0
$$

where $\mathfrak{S}_{6}$ acts by permuting the coordinates. In other words,

$$
{\overline{M_{2}(2)}}^{i n v}=\operatorname{Proj}\left(k\left[t_{0}, \ldots, t_{5}\right] /\left(s_{1}, s_{3}\right)\right) .
$$

Together with (7.1) this implies the existence of a natural compactification ${\overline{S_{2}^{+}}}^{i n v}$, the invariant-theoretical compactification of $S_{2}^{+}$, given by

$$
{\overline{S_{2}^{+}}}^{i n v}=\operatorname{Proj}\left(\left(k\left[t_{0}, \ldots, t_{5}\right] /\left(s_{1}, s_{3}\right)\right)^{G}\right) .
$$

Here $\left(k\left[t_{1}, \ldots, t_{5}\right] /\left(s_{1}, s_{3}\right)\right)^{G}$ denotes the ring of invariants in $\mathbb{C}\left[t_{0}, \ldots, t_{5}\right] /\left(s_{1}, s_{3}\right)$ where the first $\mathfrak{S}_{3}$ acts by permuting $t_{0}, t_{1}, t_{2}$, the second $\mathfrak{S}_{3}$ by permuting $t_{3}, t_{4}, t_{5}$ and $\tau$ by exchanging $t_{i}$ and $t_{i+3}$ for $i=0,1,2$.

The canonical map $M_{2}(2) \rightarrow M_{2}$ factorizes as

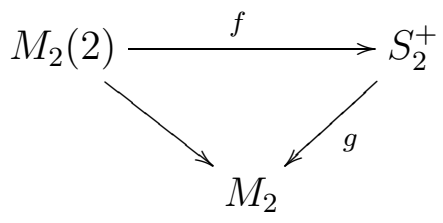

where $g$ is étale of degree 10 . If we denote by $s_{k}=s_{k}\left(t_{0}, \ldots, t_{5}\right):=\sum_{j=0}^{5} t_{j}^{k}$ for $k=$ $1, \ldots, 6$, the corresponding rings of invariants are

$$
k\left[t_{0}, \ldots, t_{5}\right] /\left(s_{1}, s_{3}\right) \supset\left(k\left[t_{0}, \ldots, t_{5}\right] /\left(s_{1}, s_{3}\right)\right)^{G} \supset\left(k\left[t_{0}, \ldots, t_{5}\right] /\left(s_{1}, s_{3}\right)\right)^{\mathfrak{S}_{6}}=k\left[s_{2}, s_{4}, s_{5}, s_{6}\right]
$$

where the last equality holds since $\mathfrak{S}_{6}$ acts by permuting the $t_{i}$. Taking the Proj of these rings gives the commutative diagram

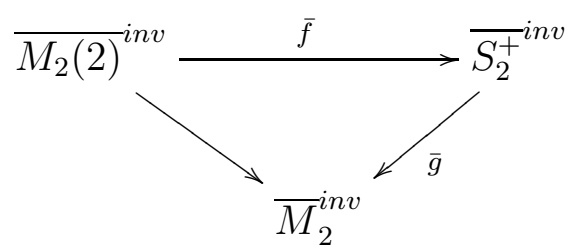

which compactifies the above diagram by (7.3) and (7.4). Note that, since the ring extensions are finite, the maps $\bar{f}$ and $\bar{g}$ are everywhere defined and finite. According to the following remark, $\bar{M}_{2}^{i n v}$ is the classical invariant theoretical compactification of $M_{2}$.

Remark 7.1. With the above coordinates we have

$$
\bar{M}_{2}^{i n v}=\operatorname{Proj}\left(k\left[s_{2}, s_{4}, s_{5}, s_{6}\right]\right)=\mathbb{P}(2,4,5,6) .
$$

On the other hand, in terms of the invariant of binary sextics (see [17] or [16]),

$$
\bar{M}_{2}^{i n v}=\operatorname{Proj}(k[A, B, C, D])=\mathbb{P}(1,2,3,5)
$$

where $A, B, C, D$ are the classical invariants degree 2,4,6,10 respectively. By [9, Proposition of Delorme] there is a natural isomorphism

$$
\mathbb{P}(2,4,5,6) \simeq \mathbb{P}(1,2,3,5) .
$$


Remark 7.2. The ring of invariants $\left(k\left[t_{0}, \ldots, t_{5}\right] /\left(s_{1}, s_{3}\right)\right)^{G}$ was explicitly computed by A. Clebsch in [6, Section 61]. For a modern version see [21, Section 4]. We do not need the explicit form of the ring.

Remark 7.3. The map $\bar{g}$ is of degree

$$
\operatorname{deg} \bar{g}=\frac{\left|S_{6}\right|}{|G|}=\frac{720}{72}=10
$$

which coincides with the fact that every smooth curve of genus 2 admits exactly 10 even theta characteristics.

In [11] the variety ${\overline{M_{2}(2)}}^{i n v}$ is interpreted as the moduli space of semistable ordered sets of 6 points in $\mathbb{P}^{1}$. As such, the strictly semistable sets are given by the 10 singular points of ${\overline{M_{2}(2)}}^{i n v}$. Here we consider ${\overline{M_{2}(2)}}^{i n v}$ as the moduli space of semistable curves of genus 2 with level-2 structure. We want to determine the image of the boundary divisor ${\overline{M_{2}(2)}}^{i n v} \backslash M_{2}(2)$ in ${\overline{S_{2}^{+}}}^{i n v}$.

Let $D_{2}(2), D_{2}^{+}$and $D_{2}$ the boundary divisors of ${\overline{M_{2}(2)}}^{i n v},{\overline{S_{2}^{+}}}^{i n v}$ and $\bar{M}_{2}^{i n v}$, i.e. $D_{2}(2)$ is the closed subscheme ${\overline{M_{2}(2)}}^{i n v} \backslash M_{2}(2)$ of ${\overline{M_{2}(2)}}^{i n v}$, etc. The above two diagrams induce the following diagram of finite surjective maps

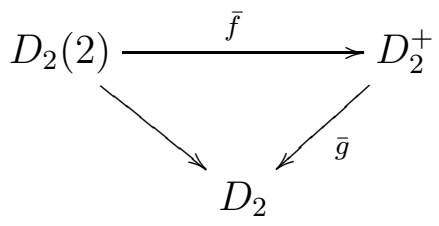

We clearly have

$$
D_{2}(2)=\Delta_{6}=\bigcup_{1 \leq i<j \leq 6} D_{i j} \quad \text { with } \quad D_{i j}=\left\{\left(x_{1}, \ldots, x_{6}\right) \in\left(\mathbb{P}^{1}\right)^{6} \mid x_{i}=x_{j}\right\} .
$$

Denoting

$$
A_{0}^{i n v}:=\bar{f}\left(D_{12}\right) \quad \text { and } \quad B_{0}^{i n v}:=\bar{f}\left(D_{14}\right)
$$

we have

Proposition 7.4. The divisor $D_{2}^{+}$consists of the 2 irreducible components

$$
D_{2}^{+}=A_{0}^{i n v} \cup B_{0}^{i n v},
$$

where $A_{0}^{\text {inv }}$ (respectively $B_{0}^{\text {inv }}$ ) is the closure of points parametrizing irreducible spin curves with one node (repectively spin curves with one exceptional component and irreducible stable reduction).

Proof. The group $G$ acts on the set of components of $D_{2}(2)$ with 2 orbits represented by $D_{12}$ and $D_{14}$ which gives the first assertion. The geometric interpretation of the components follows from [20] as in (5.1) and (5.2) above. This explains also the notation. 
According to [10, remark after Theorem 9.4.10, p.526] the boundary divisor $D_{2}(2)$ of ${\overline{M_{2}(2)}}^{i n v}=\operatorname{Proj}\left(k\left[t_{0}, \ldots, t_{5}\right) /\left(s_{1}, s_{3}\right)\right)$ is given by the 15 planes in the Segre cubic with equations

$$
t_{i}+t_{j}=t_{k}+t_{l}=t_{m}+t_{n}=0
$$

where $\{i, j, k, l, m, n\}=\{1, \ldots, 6\}$. The group $G$ acts on them with 2 orbits represented by the planes $\Pi_{1}$ with equations $t_{0}+t_{3}=t_{1}+t_{4}=t_{2}+t_{5}=0$ and $\Pi_{2}$ with equations $t_{0}+t_{1}=t_{2}+t_{3}=t_{4}+t_{5}=0$. The orbit of $\Pi_{1}$ consists of 6 planes, whereas the orbit of $\Pi_{2}$ consists of 9 planes. Comparing with (5.1) and (5.2) this implies

$$
f^{*}\left(A_{0}^{i n v}\right)=\operatorname{Orb}_{G}\left(\Pi_{1}\right) \text { and } f^{*}\left(B_{0}^{i n v}\right)=\operatorname{Orb}_{G}\left(\Pi_{2}\right)
$$

or equivalently

$$
A_{0}^{i n v}=f\left(\Pi_{1}\right) \quad \text { and } \quad B_{0}^{i n v}=f\left(\Pi_{2}\right)
$$

since the natural map $\overline{M_{2}(2)} \rightarrow{\overline{M_{2}(2)}}^{i n v}$ is G-equivariant.

The Segre cubic threefold $\bar{M}_{2}(2)$ inv contains exactly 10 singular points i.e. nodes (see [11, Example 2 p.31]). Their coordinates are $( \pm 1, \ldots, \pm 1)$ where exactly half of them are positive. Here the equation is taken in the $\mathfrak{S}_{6}$-invariant form (7.2). Denoting

$$
a_{1}^{i n v}:=\bar{f}(1,1,-1,1,-1,-1) \quad \text { and } \quad b_{1}^{i n v}:=\bar{f}(1,1,1,-1,-1,-1) .
$$

we have

Proposition 7.5. The point $a_{1}^{i n v}$ (respectively $b_{1}^{i n v}$ ) in ${\overline{S_{2}^{+}}}^{i n v}$ represents all stable spin curves with 2 smooth genus-1 components connected by one exceptional component with even (respectively odd) theta characteristics on the elliptic curves.

Proof. The group $G$ acts on 10 singular points of ${\overline{M_{2}(2)}}^{i n v}$ with orbits represented by $(1,1,-1,1,-1,-1)$ and $(1,1,1,-1,-1,-1)$. The orbit of $(1,1,-1,1,-1,-1)$ consists of 9 singular points whereas $(1,1,1,-1,-1,-1)$ is a fixed point under the action. Comparing with (5.3) and (5.4) and the definition of $A_{1}$ and $B_{1}$ gives the assertion.

$$
\text { 8. THE MAP } \bar{S}_{2}^{+} \rightarrow{\overline{S_{2}^{+}}}^{i n v}
$$

We have constructed two compactifications of the moduli space $S_{2}^{+}$of smooth even spin curves of genus 2 , namely the moduli space $\bar{S}_{2}^{+}$of generalized even spin curves of genus 2 and the invariant theoretical compactification ${\overline{S_{2}^{+}}}^{i n v}$. They fit into the following diagram

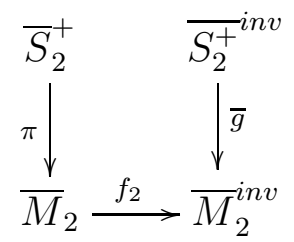

where $\pi$ denotes the forgetful map, $\bar{g}$ the map of diagram (7.5), and $f_{2}$ is the canonical holomorphic map constructed in [4, Theorem 1.1]. 
Proposition 8.1. There is a canonical holomorphic map $\overline{f_{2}}: \bar{S}_{2}^{+} \rightarrow{\overline{S_{2}^{+}}}^{i n v}$ making the following diagram commutative:

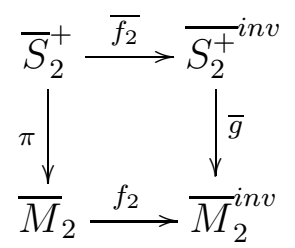

Moreover, $\overline{f_{2}}$ contracts the divisor $A_{1}$ to the point $a_{1}^{i n v}$, the divisor $B_{1}$ to the point $b_{1}^{i n v}$, and is biholomorphic on $\bar{S}_{2}^{+} \backslash\left(A_{1} \cup B_{1}\right)$.

Proof. Both spaces $\bar{S}_{2}^{+}$and ${\overline{S_{2}^{+}}}^{i n v}$ are compactifications of $S_{2}^{+}$, which gives a canonical birational map $\overline{f_{2}}$ making (8.1) commutative. The boundary of $\bar{S}_{2}^{+}$consists of the divisors $A_{0}, B_{0}, A_{1}, B_{1}$ and the boundary of ${\overline{S_{2}^{+}}}^{i n v}$ consists of the divisors $A_{0}^{i n v}, B_{0}^{i n v}$ and the points $a_{1}^{i n v}, b_{1}^{i n v}$. The spaces $A_{0} \backslash\left(A_{1} \cup B_{1}\right)$ and $A_{0}^{i n v} \backslash\left(a_{1}^{i n v} \cup b_{1}^{i n v}\right)$ (respectively $B_{0} \backslash\left(A_{1} \cup B_{1}\right)$ and $\left.B_{0}^{i n v} \backslash\left(a_{1}^{i n v} \cup b_{1}^{i n v}\right)\right)$ parametrize the same objects. Hence the map $\overline{f_{2}}$ extends to them.

On the other hand, the divisors $A_{1}$ and $B_{1}$ lie over the boundary divisor $\Delta_{1}$ of $\bar{M}_{2}$ whereas the points $a_{1}^{i n v}$ and $b_{1}^{i n v}$ lie over the boundary point $p_{s s}$ (see [3, Corollary 5.3]) of $\bar{M}_{2}^{i n v}$. Since $f_{2}$ contracts $\Delta_{1}$ to $p_{s s}$, the last assertion follows from Proposition 7.5 ,

\section{Proof of part (2) AND (3) of Theorem 2}

For the sake of abbreviation let $Y:={\overline{S_{2}^{+}}}^{i n v}$ and $X:=\bar{M}_{2}^{i n v}$ be the coarse moduli spaces and we denote by $\tilde{q}: \mathcal{Y} \rightarrow Y$ and $q: \mathcal{X} \rightarrow X$ the corresponding moduli stacks. We have the following commutative diagram:

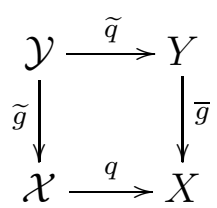

where $\bar{g}$ denotes the forgetful map of diagram 8.1 and $\widetilde{g}$ the corresponding map on the stack level. Recall from Proposition 7.4 that the boundary of $Y$ is $D_{2}^{+}=A_{0}^{i n v} \cup B_{0}^{i n v}$. Similarly the boundary of $\mathcal{Y}$ is $\mathcal{D}_{2}^{+}=\alpha_{0}^{\text {inv }}+\beta_{0}^{\text {inv }}$.

Since $\operatorname{Pic}_{\mathbb{Q}}\left(\bar{S}_{2}^{+}\right)$is generated by $A_{0}, B_{0}, A_{1}, B_{1}$ with one relation (5.5), we deduce by means of Proposition 8.1 that $\operatorname{Pic}(Y)$ is generated by the classes $A_{0}^{i n v}$ and $B_{0}^{i n v}$ with the relation

$$
3 A_{0}^{i n v}=2 B_{0}^{i n v} .
$$

It follows from Lemma 6.2 and Proposition 8.1 that the map $\bar{g}: Y \rightarrow X$ is simply ramified in $B_{0}^{i n v}$ and unramified elsewhere in codimension 1. It is well known that $\operatorname{Pic}_{\mathbb{Q}}(X)$ is generated by the class of the boundary $\Delta_{0}$. Hence

$$
\bar{g}^{*}\left(\Delta_{0}\right)=A_{0}^{i n v}+2 B_{0}^{i n v} .
$$


Further we deduce from Lemma 6.1 and the fact that the bielliptic locus of $X$ is given by the class $3 \Delta_{0}$ (see [16]), that the bielliptic locus of $Y$ is given by the class

$$
\widetilde{E}=\frac{2}{5} \cdot 3 \bar{g}^{*}\left(\Delta_{0}\right)=\frac{24}{5} A_{0}^{i n v} .
$$

As for Corollary 6.4 we obtain the following formula for $\widetilde{q}: \mathcal{Y} \rightarrow Y$. For every $\epsilon \in \mathbb{Q}$

$$
K_{\mathcal{Y}}+\epsilon \mathcal{D}_{2}^{+}=\widetilde{q}^{*}\left(\pi^{*} K_{X}+B_{0}+\frac{1}{2} \widetilde{E}+\epsilon\left(D_{2}^{+}\right)\right)
$$

holds. Using the relation (9.1) and noting that according to [16] we have

$$
K_{X}=-\frac{11}{5} \Delta_{0}
$$

we get the following ramification formula for $\widetilde{q}: \mathcal{Y} \rightarrow Y$.

Proposition 9.1. For every $\in \in \mathbb{Q}$ we have

$$
K_{\mathcal{Y}}+\epsilon\left(\mathcal{D}_{2}^{+}\right)=\tilde{q}^{*}\left(\left(-\frac{49}{10}+\frac{5}{2} \epsilon\right) A_{0}^{\text {inv }}\right) .
$$

Proof of parts (2) and (3) of Theorem 2. Proposition 9.1 implies that the class $\left(-\frac{49}{10}+\right.$ $\left.\frac{5}{2} \epsilon\right) A_{0}^{i n v}$ is nef (respectively ample) if and only if $\epsilon \geq \frac{49}{25}$ (respectively $\epsilon>\frac{49}{25}$ ).

It remains to check the singularity conditions of $Y$. Note first that the boundary of $Y$ is $A_{0}^{i n v} \cup B_{0}^{i n v}$. Moreover, the divisors $A_{0}$ and $B_{0}$ intersect non-trivially in $\bar{S}_{2}^{+}$, Proposition 8.1 implies that the contraction points $a_{1}^{i n v}$ and $b_{1}^{i n v}$ are contained in $A_{0}^{i n v} \cup B_{0}^{i n v}$. Hence the proof is the same as in the proof of part (1) of Theorem 2 in Section 6.

\section{Proof of part (2) of Theorem 3}

Recall that $\alpha$ is a canonical isomorphism $\alpha: \mathfrak{S}_{3} \widehat{M}_{2} \rightarrow S_{2}^{+}$and $\mathfrak{S}_{3} \widetilde{M}_{2}$ the partial compactification $\mathfrak{S}_{3} \widetilde{M}_{2}=\mathfrak{S}_{3} \widehat{M}_{2} \sqcup S$ of [23], with $S$ the closed subscheme of ${ }_{\mathfrak{S}_{3}} \widetilde{M}_{2}$ consisting of reducible $\mathfrak{S}_{3}$-coverings. In Section 4 we proved that the construction of the map $\alpha$ does not extend to a holomorphic map $\mathfrak{S}_{3} \widetilde{M}_{2} \rightarrow \bar{S}_{2}^{+}$. However, we show the following

Proposition 10.1. The map $\alpha: \mathfrak{S}_{3} \widehat{M}_{2} \rightarrow S_{2}^{+}$extends to a holomorphic map $\bar{\alpha}: \mathfrak{S}_{3} \widetilde{M}_{2} \rightarrow$ ${\overline{S_{2}^{+}}}^{i n v}$ which contracts the divisor $S$ to the point $a_{1}^{i n v}$ in $S_{2}^{+i n v}$.

Proof. According to Lemma 4.1 the map $\alpha$ extends to a birational map $\tilde{\alpha}: \mathfrak{S}_{3} \widetilde{M}_{2} \rightarrow S_{2}^{+}$ induced by the construction in Section 3 defined on $\mathfrak{S}_{3} \widehat{M}_{2} \cup U$ with $U \subset S$ a non-empty open set. Since the image of the the map $\alpha$ degenerates to a product of elliptic curves when a covering in $\mathfrak{S}_{3} \widehat{M}_{2}$ degenerates to a spin curve in $S$, i.e. with underlying reducible curve, the image $\tilde{\alpha}(U)$ is contained in $A_{1} \cup B_{1}$.

Consider $\left(X_{t}, \kappa_{t}\right) \in S_{2}^{+}$a family of even spin smooth curves in the image of $\alpha$ degenerating to an admissible covering $X_{0}=C_{1} \cup C_{2}$ with $C_{1}, C_{2}$ elliptic curves and $C_{1} \cap C_{2}=\{p\}$ in the following way. If $\kappa_{t}=\mathcal{O}_{X_{t}}\left(\omega_{1, t}-\omega_{2, t}+\omega_{3, t}\right)$ for some Weierstrass points $\omega_{i, t}$, $i=1,2,3$, then each element in the family comes with a $6: 1$ map $\psi_{t}: X_{t} \rightarrow \mathbb{P}^{1}$ given by the pencil $\left\langle 2 \omega_{1, t}+2 \omega_{2, t}+2 \omega_{3, t}, 2 \omega_{4, t}+2 \omega_{5, t}+2 \omega_{6, t}\right\rangle \subset\left|3 K_{X_{t}}\right|$ (see Section 3). The Weierstrass points $\omega_{i, t}$ specialize to points $\omega_{i, 0}$ lying on the component $C_{1}$ for $i=1,2,3$ and on $C_{2}$ for $i=4,5,6$. One checks that such admissible covering $X_{0} \rightarrow \mathbb{P}^{1} \cup \mathbb{P}^{1}$ 
must be totally ramified at the node $p$, which is the neutral element for both elliptic curves and the 6:1 map $C_{1} \rightarrow \mathbb{P}^{1}$ (respectively $C_{2} \rightarrow \mathbb{P}^{1}$ ) is determined by the pencil $\left\langle 2 \omega_{1,0}+2 \omega_{2,0}+2 \omega_{3,0}, 6 p\right\rangle$ (respectively $\left\langle 2 \omega_{4,0}+2 \omega_{5,0}+2 \omega_{6,0}, 6 p\right\rangle$ ). Then the theta characteristic $\kappa_{t}$ specializes to $\kappa_{0}=\left(\kappa_{C_{1}}, \kappa_{C_{2}}\right)$ where $\kappa_{C_{1}}=\mathcal{O}_{C_{1}}\left(\omega_{1,0}-\omega_{2,0}+\omega_{3,0}-p\right)$ and $\kappa_{C_{2}}=\mathcal{O}_{C_{2}}\left(\omega_{4,0}-\omega_{5,0}+\omega_{6,0}-p\right)$. Clearly these 2-torsion points are non-trivial, so $\left(X_{0}, \kappa_{0}\right) \in A_{1}$. We conclude that $\tilde{\alpha}(U) \subset A_{1}$.

Since $A_{1}$ is contracted to the point $a_{1}^{i n v}$ in ${\overline{S_{2}}}^{i n v}$ under the map $\bar{f}_{2}$, there exists a map $\bar{\alpha}$ well defined on ${ }_{\mathfrak{S}_{3}} \widetilde{M}_{2}$ making commutative the following diagram:

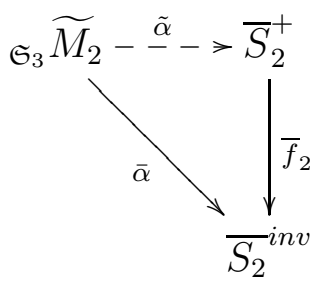

and such that $\bar{\alpha}(S)=\left\{a_{1}^{i n v}\right\}$.

\section{REFERENCES}

[1] D. Abramovich, A. Corti, A. Vistoli: Twisted bundles and admissible covers. Comm. Algebra 31 (2003), 3547-3618.

[2] E. Arbarello, M. Cornalba, P. A. Griffiths: Geometry of algebraic curves, Volume II. Grundlehren der Math. Wiss. 268, Springer - Verlag (2010)

[3] D. Avritzer, H. Lange: Pencils of quadrics, binary forms and hyperelliptic curves. Comm. in Algebra 28 (2000), 5541-5561.

[4] D. Avritzer, H. Lange: The moduli spaces of hyperelliptic curves and binary forms. Math. Z. 242 (2002), 615-632.

[5] A. Beauville: Prym varieties and the Schottky problem. Inv. Math. 41 (1977), 149-196.

[6] A. Clebsch: Theorie der binaren algebraischen Formen. Teubner, Leipzig (1872).

[7] L. Caporaso: A compactification of the universal Picard variety over the moduli space of stable curves. J. Am. Math. Soc. 7 (1994), 589-660.

[8] M. Cornalba: Moduli of curves and theta characteristics. in: Lectures on Riemann Surfaces (Trieste 1987), World Sci. Publ., 560-589.

[9] I. Dolgachev: Weighted projective varieties. Springer Lect. Notes in Math. 956 (1982), 34-71.

[10] I. Dolgachev: Classical algebraic geometry: a modern view. Cambridge University Press (2012).

[11] I. Dolgachev, D. Ortland: Point sets in projective spaces and theta functions. Astérisque 165 (1988).

[12] G. Farkas: The birational type of the moduli space of even spin curves. Advances in Math. 223 (2010), 433-443.

[13] G. Farkas A. Gibney: The Mori cone of moduli spaces of pointed curves of small genus. Trans. of the American Math. Soc. 355 (2003), 1183-1199.

[14] P. A. Griffiths, J. Harris: Principles of Algebraic Geometry. John Wiley \& Sons (1978).

[15] A. Grothendieck: Éléments de géométrie algébrique II. Publ. Math. IHES 8 (1961).

[16] B. Hassett: Classical and minimal models of the moduli space of curves of genus two. In: Geometric methods in algebra and number theory, Birkhäuser Boston (2005), 169-192.

[17] J. Igusa: On Siegel modular forms of genus two Amer. J. Math. 84 (1962), 175-200.

[18] S. Keel, J. McKernan: Contractible extremal rays in $\bar{M}_{0, n}$. In: Handbook of Moduli Vol. II, G. Farkas, I. Morrison Eds., Advanced Lect. in Math. 25, International Press (2013), 115-130.

[19] J. Kollár: Flips and abundance for algebraic threefolds. Astérisque 211 (1992). 
[20] S. Krug, Geometry of moduli spaces of spin and prym curves of small genus, Thesis, Hannover Universität (2012).

[21] V. Krishnamoorthy, T. Shaska, H. Völklein: Invariants of binary forms. arXiv:1209.0446v1 (2012).

[22] H. Lange, A. Ortega: Prym varities of triple coverings. Int. Math. Res. Notices 22 (2011), 5045-5075.

[23] H. Lange, A. Ortega: Compactification of the Prym map for non cyclic triple coverings. Int. J Math. 24, No. 3, 1350015 (2013).

[24] D. Mumford: Tata Lectures on Theta II, Progress in Math. 43, Birkhäuser (1984).

H. Lange, Department Mathematik der Universität Erlangen, Germany

E-mail address: lange@mi.uni-erlangen.de

A. Ortega, Institut für Mathematik, Humboldt Universität Zu Berlin, Germany

E-mail address: ortega@math.hu-berlin.de 